ARTICLE

Received 7 May 2015 | Accepted 18 Mar 2016 | Published 25 Apr 2016

DOI: $10.1038 /$ ncomms11386

OPEN

\title{
The HSP70 co-chaperone DNAJC14 targets misfolded pendrin for unconventional protein secretion
}

Jinsei Jung ${ }^{1,2, \star}$, Jiyoon Kim ${ }^{1, \star}$, Shin Hye Roh${ }^{1}$, Ikhyun Jun$^{1}$, Robert D. Sampson ${ }^{1}$, Heon Yung Gee ${ }^{1,3}$, Jae Young $\mathrm{Choi}^{2} \&$ Min Goo Lee ${ }^{1}$

Mutations in SLC26A4, which encodes pendrin, are responsible for hearing loss with an enlarged vestibular aqueduct and Pendred syndrome. The most prevalent mutation in East Asia is p.H723R (His723Arg), which leads to defects in protein folding and cell-surface expression. Here we show that H723R-pendrin can be rescued to the cell surface by an HSP70 co-chaperone DNAJC14-dependent unconventional trafficking pathway. Blockade of ER-to-Golgi transport or activation of ER stress signals induced Golgi-independent cell-surface expression of $\mathrm{H} 723 \mathrm{R}$-pendrin and restored its cell-surface $\mathrm{Cl}^{-} / \mathrm{HCO}_{3}^{-}$exchange activity. Proteomic and short interfering RNA screenings with subsequent molecular analyses showed that Hsc70 and DNAJC14 are required for the unconventional trafficking of H723R-pendrin. Moreover, DNAJC14 upregulation was able to induce the unconventional cell-surface expression of H723R-pendrin. These results indicate that Hsc70 and DNAJC14 play central roles in ER stress-associated unconventional protein secretion and are potential therapeutic targets for diseases such as Pendred syndrome, which arise from transport defects of misfolded proteins.

\footnotetext{
${ }^{1}$ Department of Pharmacology and Brain Korea 21 Project for Medical Sciences, Yonsei University College of Medicine, Seoul 120-752, Republic of Korea. ${ }^{2}$ Department of Otorhinolaryngology, Yonsei University College of Medicine, Seoul 120-752, Republic of Korea. ${ }^{3}$ Division of Nephrology, Department of Medicine, Boston Children's Hospital and Harvard Medical School, Boston, Massachusetts 02115, USA. * These authors contributed equally to this work. Correspondence and requests for materials should be addressed to M.G.L. (email: mlee@yuhs.ac).
} 
$\mathrm{P}$ endrin encoded by the SLC26A4 gene is a transmembrane protein that transports monovalent anions, such as $\mathrm{Cl}^{-}$, $\mathrm{I}^{-}, \mathrm{HCO}_{3}^{-}$, and formate in the inner ear, thyroid follicles and renal cortical collecting ducts ${ }^{1}$. Mutations in SLC26A4 cause non-syndromic recessive deafness with an enlarged vestibular aqueduct (DFNB4, [MIM 600791]) and Pendred syndrome (PDS, [MIM 274600]), and are considered to be the most common cause of hereditary hearing loss in East Asia and the second most common cause worldwide ${ }^{2-7}$. Specifically, p.H723R (His723Arg) is the most prevalent pathological mutation in DFNB4 and PDS, representing about $60 \%$ of presentations in Korea and Japan 8,9 . The H723R mutation results in protein misfolding, retention in the endoplasmic reticulum (ER), and finally, degradation by the ER-associated degradation (ERAD) pathway ${ }^{10}$. Consequently, negligible amounts of H723R-pendrin reach the plasma membrane, and most of the $\mathrm{Cl}^{-} / \mathrm{HCO}_{3}^{-}$or $\mathrm{Cl}^{-} / \mathrm{I}^{-}$exchange activity at the cell surface is lost.

The folding of secretory and transmembrane proteins is monitored by the ER quality control (ERQC) systems. During ERAD, non-native or misfolded proteins that do not pass ERQC are retrotranslocated to the cytoplasm and degraded by the ubiquitin-proteasome machinery 11 . Molecular chaperones and associated factors play critical roles in the recognition of ERAD target substrates. The heat shock cognate protein 70 (Hsc70; encoded by HSPA8), a member of heat shock $70 \mathrm{kDa}$ proteins (HSP70s), is one of the premier chaperones involved in this process. Although Hsc70 is a cytosolic chaperone, because most membrane proteins have large cytosolic domains, Hsc70 binds to and censors the folding status of substrate membrane proteins that are synthesized in the ER and transported to the cell surface via the conventional ER-to-Golgi secretion pathway. For example, Hsc70 has been shown to promote folding of the cystic fibrosis transmembrane conductance regulator (CFTR) $\mathrm{Cl}^{-}$channel and also to be involved in the ERAD of the folding-deficient Phe508deleted $(\Delta \mathrm{F} 508) \mathrm{CFTR}$, the most common cystic fibrosis-causing mutation worldwide ${ }^{12}$.

The HSP70 chaperones do not function alone. In fact, cochaperones of Hsc70, such as Hdj-2 and CHIP, have been shown to participate in the folding, biogenesis and degradation of CFTR $^{13,14}$. Notably, the HSP70 proteins not only are involved in protein folding and degradation but also function in a myriad of biological processes, including protein-protein interaction and intracellular trafficking of various proteins ${ }^{15,16}$. This multitude of roles is not easily reconciled with the simple activity of HSP70s in ATP-dependent substrate protein binding and release cycles. Consequently, it has been suggested that much of the functional diversity of HSP70 is driven by a diverse class of cofactors, such as J proteins, also known as HSP40s (ref. 15).

Recently, it was found that $\Delta$ F508-CFTR can be expressed on the cell surface via an unconventional secretion pathway under specific cellular conditions ${ }^{17}$. Blockade of ER-to-Golgi transport induces ER stress and unfolded protein response due to accumulation of excessive nascent, unfolded polypeptides in the $\mathrm{ER}^{18}$. Interestingly, ER-to-Golgi blockade or activation of ER stress signals induced the plasma membrane expression of $\Delta$ F508-CFTR via a Golgi-bypass trafficking pathway and restored CFTR $\mathrm{Cl}^{-}$channel activity at the cell surface ${ }^{17}$. Although Golgi reassembly and stacking proteins (GRASPs) were identified as a cargo-recruiting factor in this unconventional transport of $\Delta$ F508-CFTR, the mechanisms underlying ER-toGolgi blockade or ER stress-associated unconventional protein secretion pathway are largely unknown.

Here we report that the clinically important, folding-deficient membrane protein H723R-pendrin can be rescued to the cell surface by the unconventional protein secretion pathway. More importantly, the HSP70 co-chaperone DNAJC14 plays an essential role in allowing H723R-pendrin to escape ERAD and be expressed on the cell surface. These results provide insights into the mechanism of ER stress-associated unconventional protein secretion. Furthermore, identification of DNAJC14 as a factor targeting entry of a folding-deficient membrane protein into the unconventional protein secretion pathway will have an impact on the development of therapeutic strategies for diseases arising from transport defects of misfolded proteins.

\section{Results}

Unconventional secretion of pendrin by ER-to-Golgi blockade. Pendrin is a glycosylated membrane protein that undergoes ER core-glycosylation (band $\mathrm{B}, \sim 84 \mathrm{kDa}$, Fig. 1a) and Golgi complex-glycosylation (band $\mathrm{C}, \sim 120 \mathrm{kDa}$ ) during conventional ER-to-Golgi exocytosis, with the non-glycosylated nascent polypeptides (band $\mathrm{A}, \sim 82 \mathrm{kDa}$ ) being barely detectable in the immunoblot of cytosolic samples. It is commonly assumed that the mature complex-glycosylated pendrin is the only form expressed in the plasma membrane ${ }^{10}$. However, in the case of CFTR, the immature ER form of core-glycosylated protein can be unconventionally expressed on the cell surface by ER-to-Golgi blockade $^{17}$. Therefore, we examined whether a similar phenomenon exists with pendrin. PANC-1 cells derived from pancreatic ducts were used to examine the cell-surface expression of pendrin. Because these cells retain epithelial properties and do not express detectable levels of endogenous pendrin, they constitute a suitable model for studying the intracellular trafficking of pendrin through transfection of plasmids encoding the mutant protein.

Inhibition of ER-to-Golgi traffic was achieved by expression of the dominant-negative forms of Arf1 (a component of the coat protein complex [COP]I) and Sar1 (a component of COPII), or by overexpression of syntaxin 5 (required for the fusion of COPII transport vesicles with the acceptor Golgi membrane $)^{17}$. As shown in Fig. 1b, when wild-type (WT) pendrin was expressed in PANC-1 cells, the band C form of complex-glycosylated pendrin was mainly expressed on the cell surface. Blockade of ER-to-Golgi transport induced by the Arf1-Q71L and Sar1-T39N dominantnegative mutants or overexpressed syntaxin 5 completely blocked the Golgi maturation of WT-pendrin glycosylation. Interestingly, even when the ER-to-Golgi transport was blocked, the band B form of core-glycosylated WT-pendrin continued to reach the plasma membrane (Fig. 1b). More importantly, the foldingdeficient H723R-pendrin, which did not travel to the cell surface in control cells, reached the cell surface when ER-to-Golgi trafficking was blocked (Fig. 1c).

Similar to the case of $\Delta \mathrm{F}^{2} 08-\mathrm{CFTR}^{17}$, ER stress-inducing agents, such as brefeldin A (BFA) and thapsigargin, also induced the cell-surface expression of core-glycosylated H723R-pendrin (Fig. 1d). Furthermore, depletion of three major ER stress-signal transducers, IRE1 $\alpha$, PERK and ATF6 (ref. 19), by treatment with specific siRNAs revealed that the IRE1 $\alpha$-mediated signalling arm is involved in Arf1-Q71L-induced exocytosis of H723R-pendrin (Supplementary Fig. 1). These results suggest that ER stressrelated signals are required for the unconventional secretion of core-glycosylated pendrins. Unlike $\Delta$ F508-CFTR, in which the unconventional cell-surface expression is dependent on GRASP $^{17}$, cell-surface expression of H723R-pendrin was neither inhibited by depletion of GRASPs (Supplementary Fig. 2a) nor induced by overexpression of GRASP55 (Fig. 1e; Supplementary Fig. 2b). As a control, GRASP55-mediated induction of cellsurface expression of $\Delta$ F508-CFTR was recapitulated in this study using PANC-1 cells (Fig. 1f; Supplementary Fig. 2c).

To determine whether the core-glycosylated H723R-pendrin transported to the cell surface was functional, the pendrin-dependent 
a

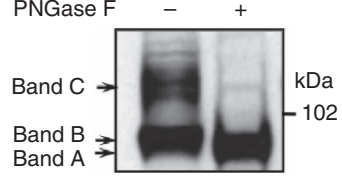

b

$\begin{array}{rllll}\text { WT-Pendrin } & + & + & + & + \\ \text { HA-Arf1-Q71L } & - & + & - & - \\ \text { Myc-Sar1-T39N } & - & - & + & - \\ \text { HA-Syntaxin 5 } & - & - & - & +\end{array}$
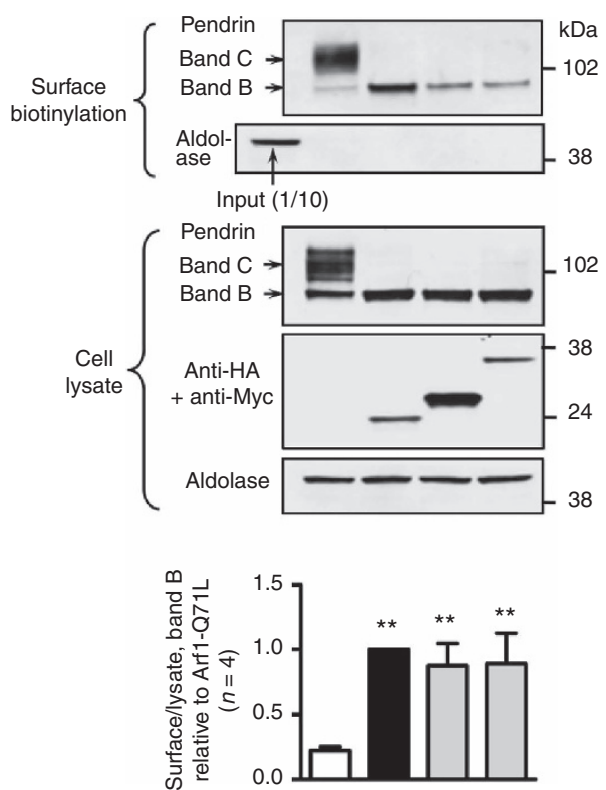

e
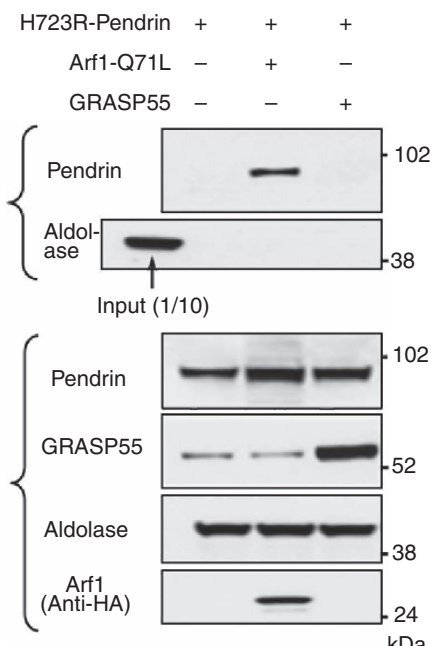

$\mathrm{kDa}$
Input (1/10)

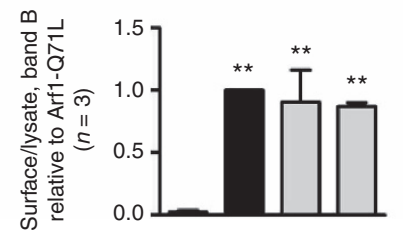

H723R-Pendrin -++++

HA-Arf1-Q71L - $\quad+\quad-\quad$

BFA $\left(5 \mu \mathrm{g} \mathrm{ml}^{-1}, 6 \mathrm{~h}\right) \quad-\quad-\quad+\quad+$

$\mathrm{TG}(1 \mu \mathrm{M}, 6 \mathrm{~h}) \quad-\quad \ldots \quad-\quad \ldots+$

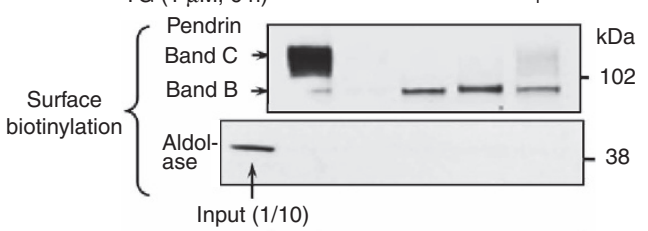

Pendrin
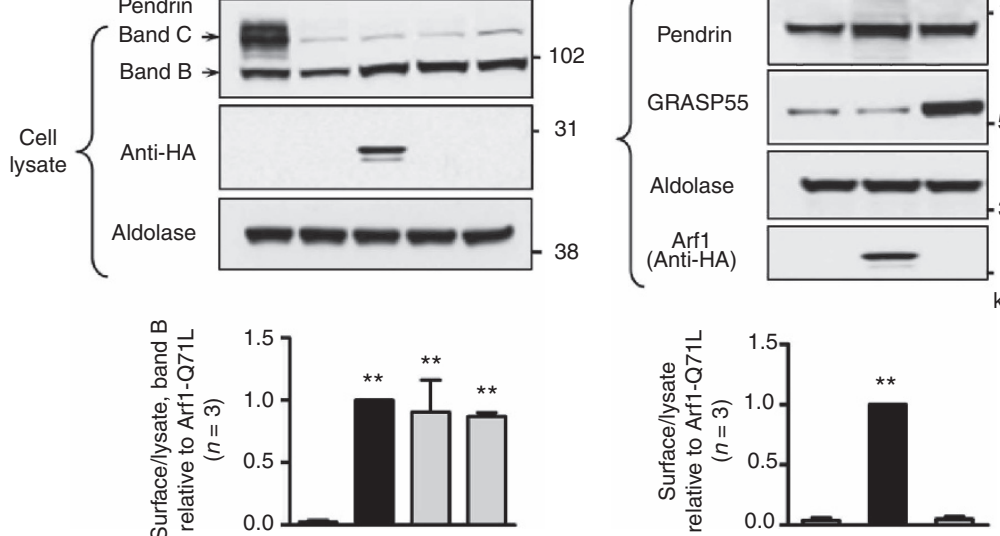

C

\begin{tabular}{|c|c|c|c|}
\hline H723R-Pendrin & + & + & + \\
\hline HA-Arf1-Q71L & - & + & \\
\hline Myc-Sar1-T39N & - & - & + \\
\hline HA-Syntaxin 5 & - & - & - \\
\hline
\end{tabular}
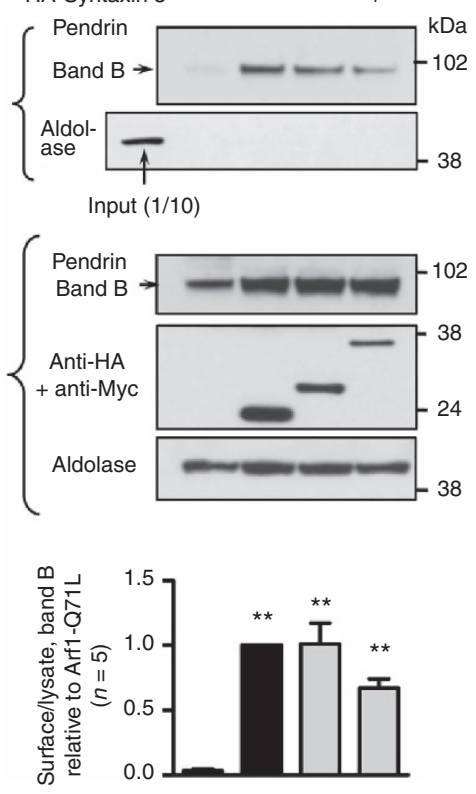

$f$

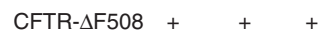

Arf1-Q71L - + -

GRASP55 - $\quad$ - +
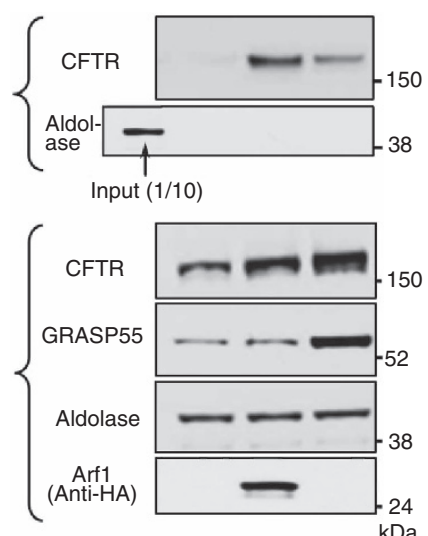

$\mathrm{kDa}$

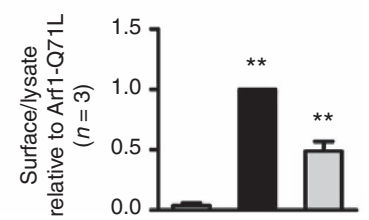

Figure 1 | Cell-surface expression of H723R-pendrin by unconventional protein secretion. (a) PANC-1 cells were transfected with plasmids encoding wildtype (WT)-pendrin. Protein samples were treated with N-glycosidase F (PNGase F), which deglycosylates all N-glycan chains and immunoblotted with antipendrin antibodies. Band A: deglycosylated pendrin, band B: immature core-glycosylated pendrin, band C: mature complex-glycosylated pendrin. (b,c) Surface biotinylation assays were performed in PANC-1 cells expressing WT- (b) or H723R- (c) pendrin. In control cells (first lanes in $\mathbf{b}$ and $\mathbf{c}$ ), the band C form of WTpendrin and no form of H723-pendrin were expressed on the cell surface. Blockade of ER-to-Golgi traffic by overexpression of Arf1-Q71L, Sar1-T39N or syntaxin 5 induced the cell-surface expression of core-glycosylated (band B) WT- and H723R-pendrins. The absence of the cytosolic protein aldolase A in the biotinylated fraction confirmed cell surface protein-specific labelling in each experiment (input: $40 \mu \mathrm{g}$ of cell lysate, surface biotinylation: $400 \mu \mathrm{g}$ protein). (d) Treatments with brefeldin A (BFA) or thapsigargin (TG) also induced cell-surface expression of H723R-pendrin in PANC-1 cells. (e,f) Whereas GRASP55 overexpression induced cell-surface expression of $\triangle$ F508-CFTR in PANC-1 cells (f), GRASP55 was not effective in inducing cell-surface expression of H723R-pendrin (e). Quantitation of multiple experiments is presented under each immunoblot. ${ }^{* \star} P<0.01$ by one-way analysis of variance, compared with lane 1 , the number of replicates $(n)$ is presented in each panel. Unprocessed original scans of western blots are shown in Supplementary Fig. 10. 

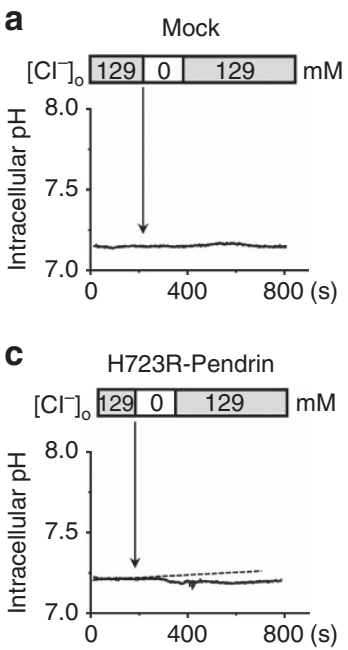

b

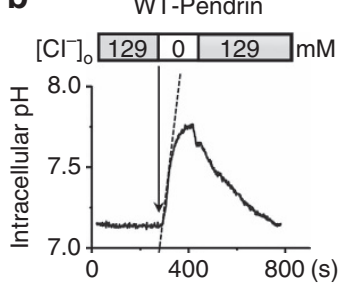

$\mathbf{d}_{\text {H723R-Pendrin }+ \text { Arf1-Q71L }}$ $\left[\mathrm{Cl}^{-}\right]_{0}\left[\begin{array}{ll|l}129 & 0 & 129 \\ \hline\end{array} \mathrm{mM}\right.$

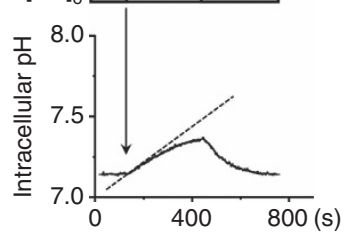

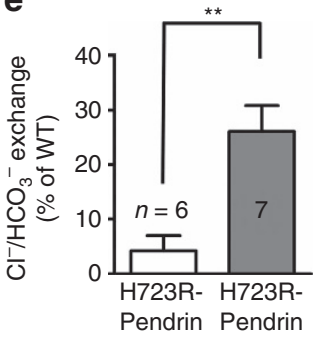

$+$

Figure 2 | Rescue of anion exchange activity of H723R-pendrin by Arf1-Q71L. The $\mathrm{Cl}^{-} / \mathrm{HCO}_{3}^{-}$exchange activity was measured by recording pH $\mathrm{H}_{\mathrm{i}}$ as detailed in Methods. A clone of HEK 293 cells that exhibited minimal intrinsic anion $\left(\mathrm{Cl}^{-} / \mathrm{HCO}_{3}^{-}\right)$exchange activity (a) was transfected with the indicated plasmids (b-d) and then anion exchange activity was analysed. The quantitation of multiple experiments is depicted in e. Blockade of ER-to-Golgi trafficking by Arf1-Q71L significantly increased $\mathrm{Cl}^{-} / \mathrm{HCO}_{3}^{-}$exchange activity in cells expressing $\mathrm{H} 723 \mathrm{R}$-pendrin. ${ }^{\star \star} P<0.01$ by unpaired Student's $t$-test, the number of replicates $(n)$ is presented in each lane.

$\mathrm{Cl}^{-} / \mathrm{HCO}_{3}^{-}$exchange activity was examined in a clone of HEK 293 cells that has minimal intrinsic $\mathrm{Cl}^{-} / \mathrm{HCO}_{3}^{-}$exchange activity (Fig. 2a-e). The $\mathrm{Cl}^{-} / \mathrm{HCO}_{3}^{-}$exchange activity was measured in cells attached to coverslips by recording $\mathrm{pH}_{\mathrm{i}}$ in response to $\left[\mathrm{Cl}^{-}\right]_{\mathrm{o}}$ changes of the perfusate. Strong $\mathrm{Cl}^{-} / \mathrm{HCO}_{3}^{-}$exchange activity was elicited by transfection with WT-pendrin $(0.230 \pm 0.029 \Delta \mathrm{pH}$ unit $\left.\min ^{-1}, n=13\right)$, but not H723R-pendrin $(0.010 \pm 0.006 \Delta \mathrm{pH}$ unit $\left.\min ^{-1}, n=6\right)$. Notably, blockade of the ER-to-Golgi transport by Arf1-Q71L significantly increased the H723R-pendrin-mediated $\mathrm{Cl}^{-} / \mathrm{HCO}_{3}^{-}$exchange activity to $0.060 \pm 0.011 \Delta \mathrm{pH}$ unit $\mathrm{min}^{-1}$ $(n=7, P<0.01)$, which corresponded to $26 \%$ of the WT-pendrin activity. Taken together, these results indicate that ER-to-Golgi blockade or ER stress induces the functional cell-surface expression of core-glycosylated H723R-pendrin via a GRASP-independent route that bypasses the Golgi.

Hsc70 is required for unconventional secretion of pendrin. To identify the components involved in the unconventional cell-surface transport of core-glycosylated pendrin, we first examined the binding partners of H723R-pendrin using tandem affinity purification (TAP) with mass spectrometry ${ }^{20}$. After electrophoretic separation of proteins bound to H723R-pendrin in lysates of cells expressing H723R-pendrin alone or together with Arf1-Q71L (Fig. 3a), each protein band was purified, and protein identities were determined by liquid chromatography-tandem mass spectrometry (LC-MS/MS). Among the high Mowse-score proteins, four candidate proteins that are relevant to ERQC or protein trafficking were selected for further investigation (Supplementary Table 1). A loss-of-function study was then performed with siRNAs against each target protein (Fig. 3b-f). Knockdown (60-80\%) of each target protein by siRNA treatment was confirmed using quantitative PCR (Supplementary Fig. 3a). Notably, knockdown of Hsc70 significantly decreased the surface expression of H723R-pendrin induced by Arf1-Q71L (Fig. 3e,f). In addition, BFA-induced unconventional trafficking of H723R-pendrin was diminished by treatment with siRNA targeting Hsc70 (Supplementary Fig. 3b-f). Interestingly, unconventionally surfacetransported $\Delta$ F508-CFTR was also reduced by knockdown of Hsc70 (Supplementary Fig. 3g). We next examined whether overexpression of the candidate genes induced the surface expression of H723Rpendrin; however, none of the candidate proteins had this effect
(Fig. 3g). These results indicate that Hsc70 is necessary, but not sufficient, for unconventional surface trafficking of H723R-pendrin.

We then further examined the role of $\mathrm{Hsc70}$ in the unconventional secretion of core-glycosylated pendrin. Immunoprecipitation results revealed that both core-glycosylated WTpendrin (Fig. 4a) and H723R-pendrin (Fig. 4b) strongly interacted with Hsc70 when ER-to-Golgi trafficking was blocked by Arf1-Q71L or syntaxin 5 overexpression. The ATPase activity of Hsc70 plays a key role in the binding and release of Hsc70 substrates ${ }^{11}$. To investigate whether the ATPase activity of Hsc70 is necessary for surface transport of core-glycosylated H723Rpendrin, the ATPase-deficient Hsc70-K71M mutant ${ }^{21}$ was overexpressed in cells or cells were treated with apoptozole, the inhibitor of Hsc70 ATPase activity ${ }^{22}$. Inhibition of the ATPase activity by Hsc70-K71M reduced the unconventional surface expression of H723R-pendrin (Fig. 4c). Similarly, apoptozole inhibited the unconventional transport of H723R-pendrin in a dose-dependent manner (Fig. 4d), indicating that the ATPase activity of Hsc70 is important for the unconventional transport of immature core-glycosylated pendrin.

DNAJC14 participates in unconventional secretion of pendrin. In the canonical model of the ATP-dependent binding and release cycle of HSP70 substrates, J proteins and nucleotide exchange factors (NEFs) are key regulators of HSP70 function ${ }^{15}$. In addition, co-chaperones have been suggested to be responsible for the functional diversity of the HSPs ${ }^{15}$. Therefore, we hypothesized that co-chaperone(s) are involved in the Hsc70-dependent unconventional trafficking of pendrin. A total of $25 \mathrm{~J}$ proteins, NEFs, HSP90 components, and other co-chaperones known to interact with Hsc70 were knocked down using a custom siRNA library, and Arf1-Q71L-mediated surface expression of H723Rpendrin was analysed to identify the Hsc70 co-chaperone associated with the unconventional secretion of immature coreglycosylated pendrin. The initial screening revealed that knockdown of DNAJC14 (a member of the J protein family also known as DRIP78, Jiv, or LIP6) had the strongest effect in reducing the unconventional secretion of H723R-pendrin (Fig. 5a and Supplementary Fig. 4). Subsequent analyses further confirmed the involvement of DNAJC14 in the unconventional secretion of 
a

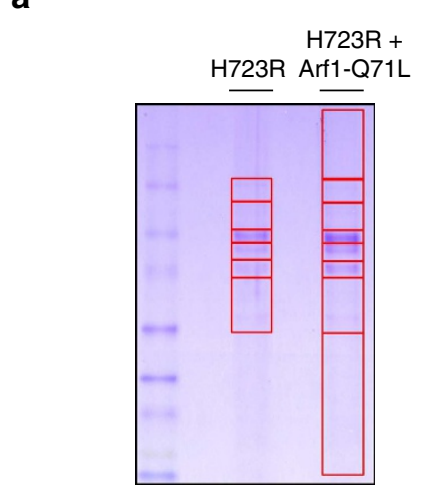

g

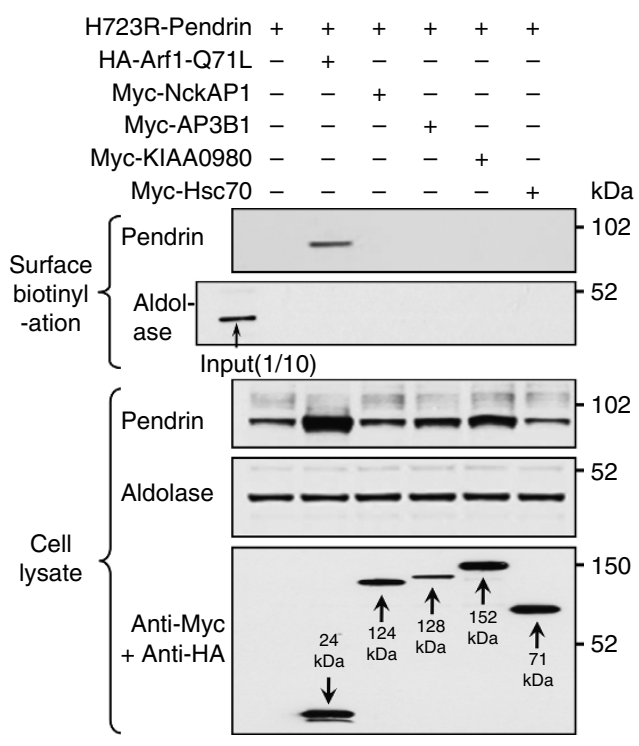

b

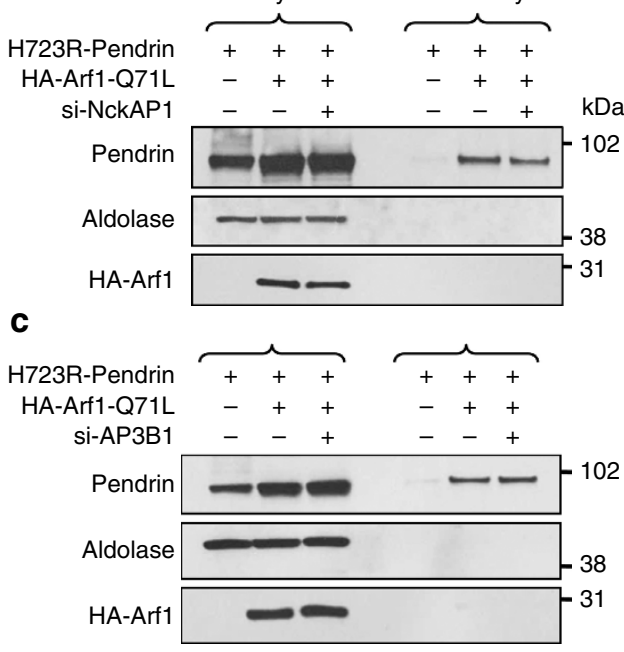

d

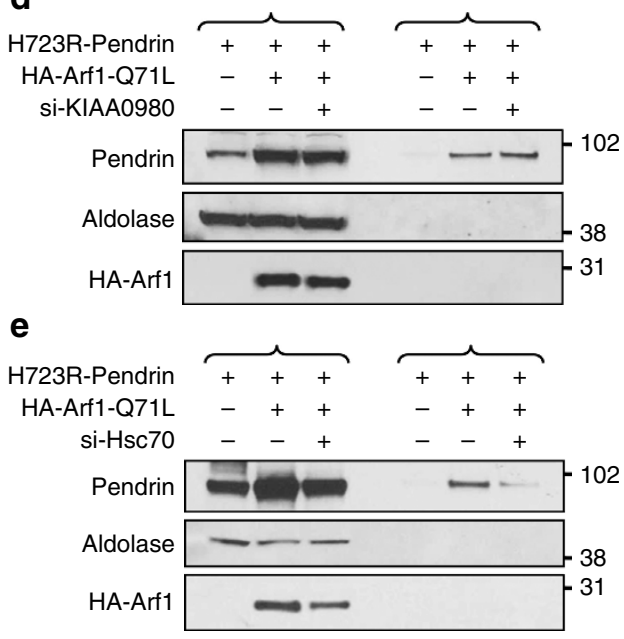

$\mathbf{f}$

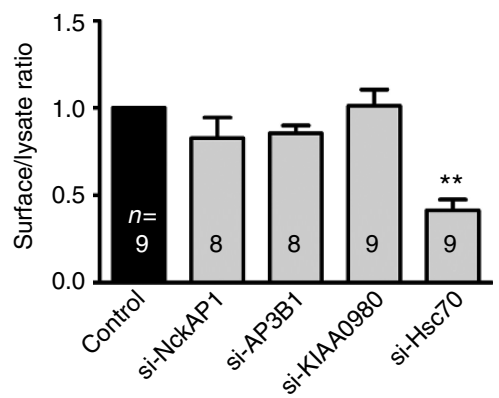

Figure 3 | Hsc70 is necessary for Arf1-Q71L-mediated unconventional cell-surface expression of H723R-pendrin. (a) After tandem affinity purification, binding partners of H723R-pendrin in PANC-1 cells with or without Arf1-Q71L co-expression were separated by electrophoresis. Subsequent analyses by LC-MS/MS identified NckAP1, AP3B1, KIAA0980 and Hsc70 as binding partners of H723R-pendrin (Supplementary Table 1). (b-f) Surface biotinylation assays were performed after siRNA-mediated knockdown of indicated candidate proteins. Representative blots are shown in $\mathbf{b}-\mathbf{e}$ and a summary of multiple experiments is presented in $\mathbf{f}$. Only treatment with siRNA targeting $\mathrm{Hsc70}$ showed a significant effect. ${ }^{\star \star} P<0.01$ by one-way analysis of variance, difference from control, the number of replicates $(n)$ is presented in each lane. (g) Surface biotinylation assays were performed after overexpression of NckAP1, AP3B1, KIAA0980 and Hsc70. None of the candidate proteins induced cell-surface expression of H723R-pendrin. The data are representative of three independent experiments. Unprocessed original scans of western blots are shown in Supplementary Fig. 10.

H723R-pendrin. When Hsc70 and DNAJC14 were simultaneously depleted, the Arf1-Q71L-induced cell-surface expression of H723R-pendrin was almost completely abolished (Fig. 5b). However, DNAJC14 was not involved in the unconventional secretion of $\Delta$ F508-CFTR (Supplementary Fig. 5), implying that substrate specificity exists in the DNAJC14-mediated unconventional secretion. Quantitative PCR analyses revealed that Arf1-Q71L or thapsigargin did not affect the endogenous levels of DNAJC14 messenger RNA in PANC-1 cells (Fig. 5c). In contrast, Arf1-Q71L evoked co-immunoprecipitation between DNAJC14 and H723R-pendrin (Fig. 5d), suggesting that a physical association with the substrate protein rather than a 
a

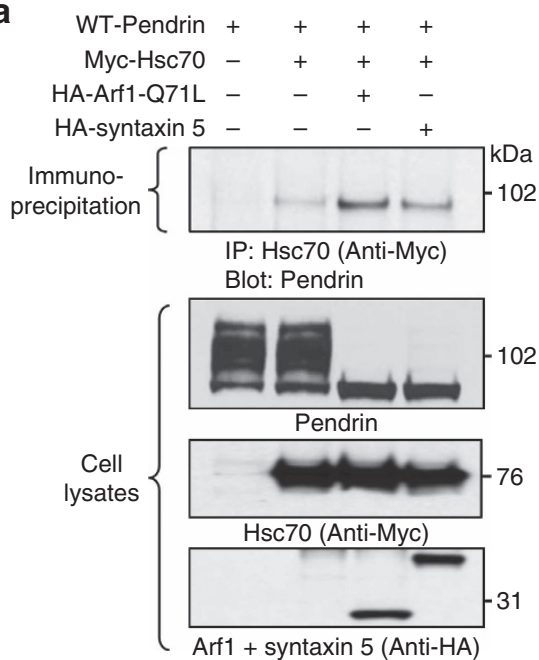

C
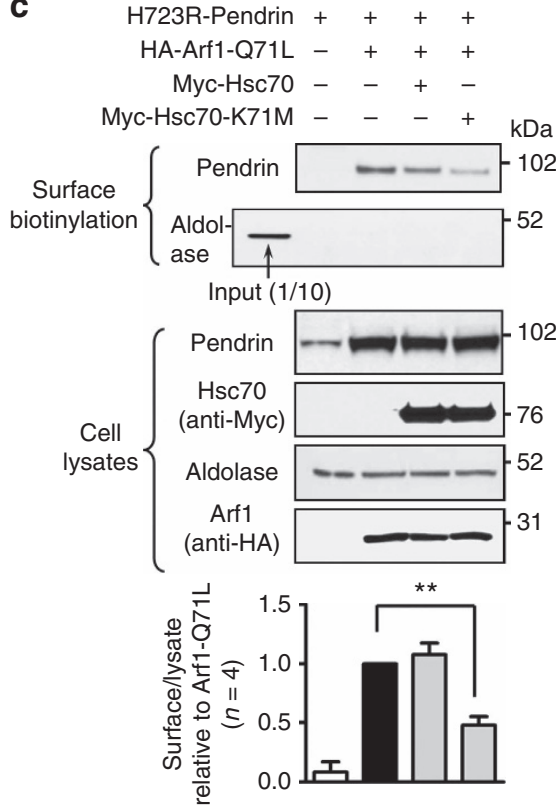

b

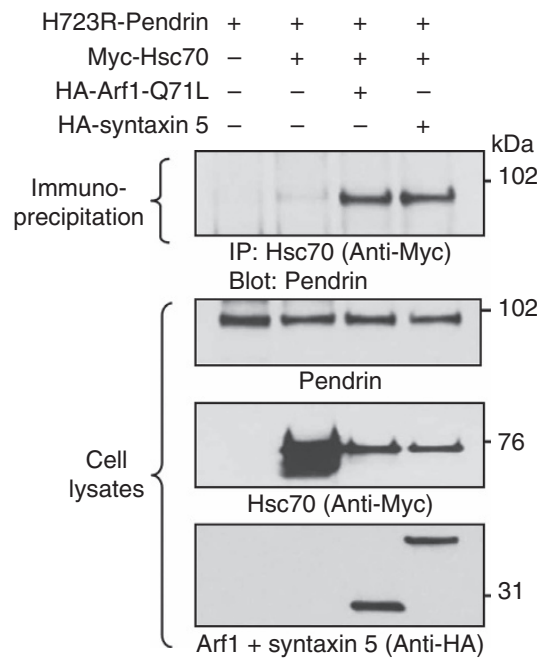

d
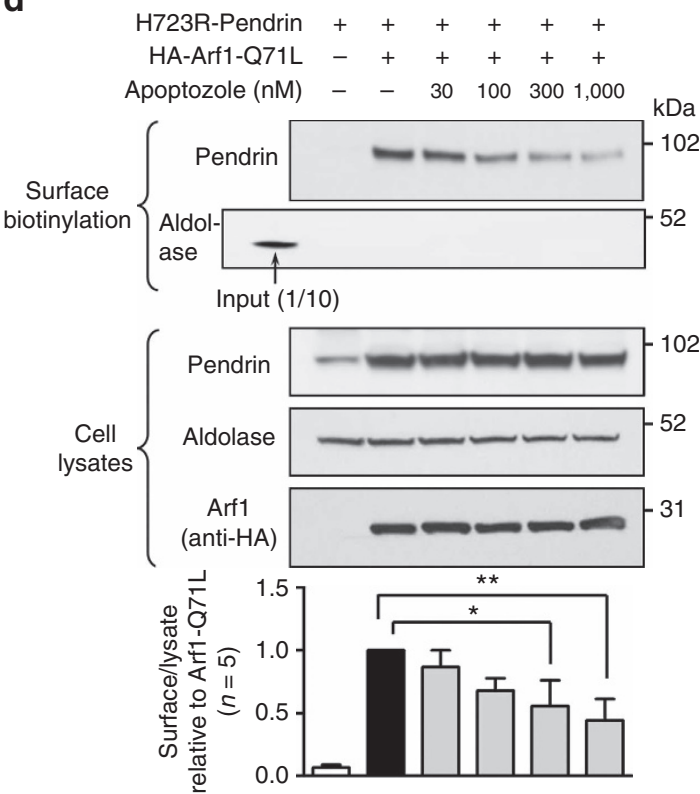

Figure 4 | Physical interaction with Hsc70 and Hsc70 ATPase activity are necessary for unconventional surface trafficking of H723R-pendrin.

$(\mathbf{a}, \mathbf{b})$ Lysates of PANC-1 cells transfected with indicated plasmids were immunoprecipitated with anti-Myc (Hsc70) and immunoblotted. Both and WT- (a) and H723R- (b) pendrins strongly interacted with Hsc70 when ER-to-Golgi traffic was blocked by Arf1-Q71L or syntaxin 5 overexpression. (c) Inhibition of Hsc70 ATPase activity by overexpression of Hsc70-K71M (ATPase activity-ablated dominant-negative mutant) inhibited the surface expression of unconventionally transported H723R-pendrin. (d) Hsc70 ATPase activity inhibitor apoptozole dose-dependently reduced Arf1-Q71L-induced surface expression of H723R-pendrin. ${ }^{\star} P<0.05,{ }^{\star \star} P<0.01$ by one-way analysis of variance, the number of replicates $(n)$ is presented in each panel. Unprocessed original scans of western blots are shown in Supplementary Fig. 10.

translational regulation of DNAJC14 underlies the mechanism of DNAJC14-mediated unconventional protein secretion.

To further substantiate our observations, we evaluated the surface expression of pendrin using immunofluorescence analysis. In permeabilized control cells (Fig. 6a), WT-pendrin was expressed at the cell surface, whereas most H723R-pendrin was located in the ER. Notably, a significant amount of H723Rpendrin reached the cell surface when ER-to-Golgi trafficking was blocked by Arf1-Q71L. Importantly, the Arf1-Q71L-induced surface expression of H723R-pendrin was abolished by treatment with siRNA targeting Hsc70 or DNAJC14 (Fig. 6a). These events were more evident in the immunostaining of non-permeabilized cells (Fig. 6b,c). All of these biochemical and morphological results indicate that $\mathrm{Hsc70}$ and DNAJC14 are necessary for the unconventional cell-surface expression of H723R-pendrin.
Rescue of H723R-pendrin trafficking by DNAJC14 upregulation. Next, we examined whether DNAJC14 upregulation can rescue the transport defects caused by the H723R-pendrin mutation. Control experiments with WT-pendrin indicated that neither overexpression of Hsc70 and DNAJC14 nor inhibition of Hsc70ATPase activity by Hsc70-K71M affected the conventional surface transport of mature WT-pendrin (Fig. 7a). In contrast, overexpression of DNAJC14 alone or together with Hsc70 induced the surface expression of H723R-pendrin (Fig. 7b) without significantly inducing ER stress (Fig. 7c). Similar to the effects induced by Arf1Q71L (Fig. 5a), DNAJC14-induced cell-surface expression of H723R-pendrin was not inhibited by the single depletion of NEFs or components of the HSP90 chaperone complex (Supplementary Fig. 6a,b). However, DNAJC14-induced surface expression of H723R-pendrin was reduced significantly by Hsc70-K71M (Fig. 7b) 

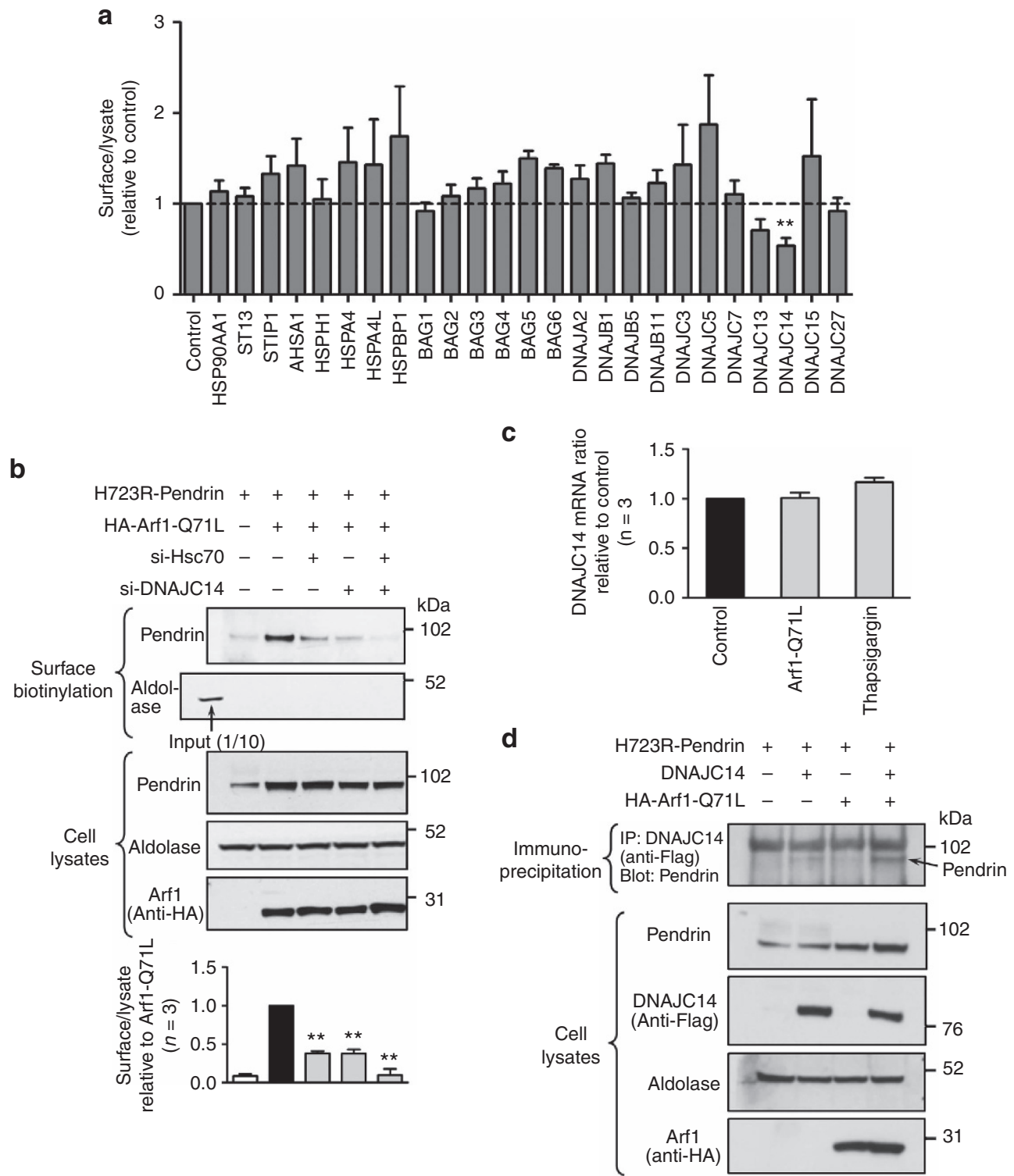

Figure 5 | DNAJC14 is necessary for unconventional surface trafficking of H723R-pendrin. (a) An siRNA screen was performed in PANC-1 cells transfected with plasmids encoding H723R-pendrin and Arf1-Q71L to identify J proteins or nucleotide exchange factors involved in the unconventional transport of $\mathrm{H} 723$-pendrin. Cells were treated with siRNA $24 \mathrm{~h}$ before transfection with plasmids. Surface biotinylation assays were performed $24 \mathrm{~h}$ after plasmid transfection, and the surface-to-lysate ratio was calculated by densitometry. A summary of multiple experiments is shown, and representative immunoblots are presented in Supplementary Fig. $4 .{ }^{* *} P<0.01$ by one-way analysis of variance (ANOVA), $n=4-7$. (b) Treatment with siRNAs targeting DNAJC14 and Hsc70 cooperatively reduced the surface expression of unconventionally transported H723R-pendrin. ${ }^{\star *} P<0.01$ by one-way ANOVA, difference from Arf1-Q71L alone (lane 2), $n=3$. (c) Quantitative PCR analyses of DNAJC14 in PANC-1 cells. Arf1-Q71L or thapsigargin did not significantly alter the mRNA levels of DNAJC14. (d) Proteins samples from PANC-1 cells transfected with indicated plasmids were immunoprecipitated with anti-Flag (DNAJC14) and immunoblotted. Arf1-Q71L-mediated ER-to-Golgi blockade induced the association of DNAJC14 with H723R-pendrin. The data are representative of three independent experiments. Unprocessed original scans of western blots are shown in Supplementary Fig. 10.

and by co-expression of other J proteins that may compete with DNAJC14 in associating with Hsc70 (Supplementary Fig. 6c), indicating that functionally competent $\mathrm{Hsc70}$ is required for DNAJC14 activity. DNAJC14 contains a tripeptide of histidine, proline, and aspartic acid residues (HPD motif), which is highly conserved among DNAJ proteins and critical for accelerating the ATPase activity of Hsc70 (refs 21,23). Notably, the H471Q mutation abolished the ability of DNAJC14 to induce the cellsurface expression of H723R-pendrin (Fig. 7d), further supporting the importance of the DNAJC14 and HSP70 interaction and the ATPase activity of HSP70 in the unconventional trafficking of pendrin.
Functional analyses showed that DNAJC14 alone increased the $\mathrm{Cl}^{-} / \mathrm{HCO}_{3}^{-}$exchange activity of H723R-expressing PANC-1 cells from $0.039 \pm 0.015 \Delta \mathrm{pH}$ unit $\mathrm{min}^{-1}$ to $0.201 \pm 0.034 \Delta \mathrm{pH}$ unit $\min ^{-1}(P<0.01)$ (Fig. 8a-e). Measurement of $\mathrm{Cl}^{-} / \mathrm{I}^{-}$exchange activity further confirmed that DNAJC14 upregulation induced the cell-surface expression of functionally active H723R-pendrin (Supplementary Fig. 7). Co-expression of Hsc70 did not significantly augment the DNAJC14 effects. However, Hsc70-K71M blocked the effects of DNAJC14, again indicating that the effect of DNAJC14 is dependent on the ATPase activity of endogenous Hsc70.

The immunostaining results were consistent with the surface biotinylation and functional data. DNAJC14, alone or together 
a

$\begin{array}{rc}\text { WT-Pendrin } & + \\ \text { H723R-Pendrin } & - \\ \text { Arf1-Q71L } & - \\ \text { SiHsc70 } & - \\ \text { siDNAJC14 } & -\end{array}$

Permeabilized

SIDNAJC14

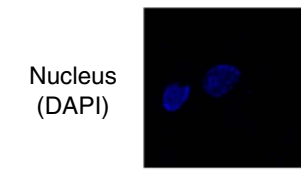

ER

ER
(Calnexin)
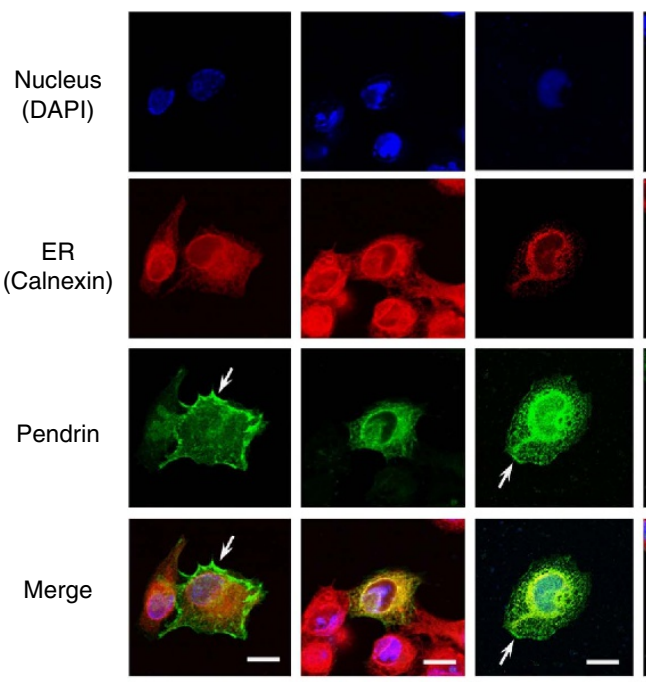

b

Non-

permeabilized
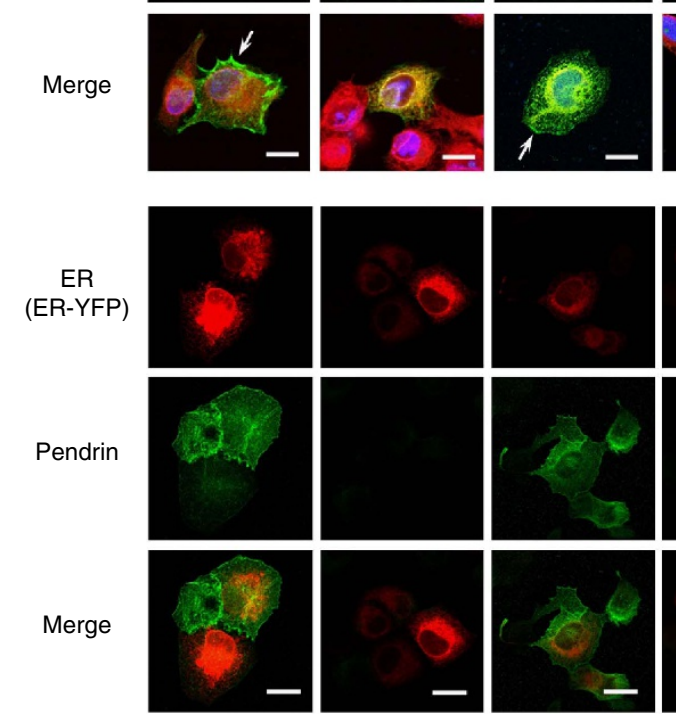

Merge
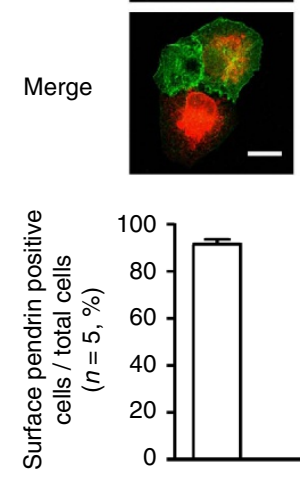

\section{c}

Non- permeabilized

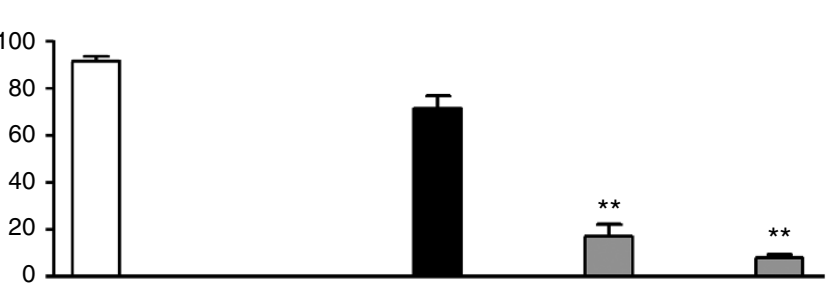

Figure 6 | Knockdown of Hsc70 and DNAJ14 inhibits the cell-surface expression H723R-pendrin induced by Arf1-Q71L. (a) Cellular localization of pendrin was examined in PANC-1 cells permeabilized with ethanol and acetone. Cells stained for WT- and H723R-pendrin were co-stained for the ER marker protein calnexin. Arrows indicate the cell-surface expression of WT- or H723R-pendrin. Nuclei were counterstained with 4',6-diamidino-2phenylindole (DAPI). Scale bar, $20 \mu \mathrm{m}$. (b,c) Cell-surface expression of WT- and H723R-pendrin was examined in non-permeabilized PANC-1 cells (4\% formaldehyde fixation) using antibodies against the extracellular $N$ terminus of pendrin ${ }^{8}$ (Abcam, ab66702). The plasmid encoding the ER marker protein ER-yellow fluorescent protein (YFP) was co-transfected. Arf1-Q71L induced the cell-surface expression of H723R-pendrin, which was blocked by siRNAs targeting Hsc70 and DNAJC14. Quantification of the ratio of cells expressing pendrin on the cell surface relative to total cells in five independent experiments is shown in c. Scale bar, $20 \mu \mathrm{m} .{ }^{* \star} \mathrm{P}<0.01$ by one-way analysis of variance, difference from H723R-Pendrin + Arf1-Q71L alone (lane 3$), n=5$.

with Hsc70, induced the cell-surface expression of H723Rpendrin in non-permeabilized cells. In addition, Hsc70-K71M blocked the DNAJC14-mediated effect and DNAJC14-H471Q was not effective in inducing surface trafficking of H723Rpendrin (Fig. 9a,b). Then, we examined the localization of DNAJC14 in membrane-permeabilized cells. Interestingly, although DNAJC14 is known to be an ER chaperone, overexpression of this protein led to its distribution both within the ER and in multiple cytosolic punctate structures, where it colocalized with Hsc70 (Fig. 10a,b). The Hsc70-K71M and DNAJC14-H471Q mutations significantly inhibited the colocalization of Hsc70 and DNAJC14 (Fig. 10a,b). In an effort to identify the punctate structures, cells were immunostained for multiple markers of organelles and vesicles. Of these, overexpressed DNAJC14 clearly colocalized with Rab18, which is considered to be a marker of lipid droplets ${ }^{24}$ (Supplementary Fig. 8). The association between DNAJC14 and Rab18-containing structures was also recapitulated on examination of endogenous 
a
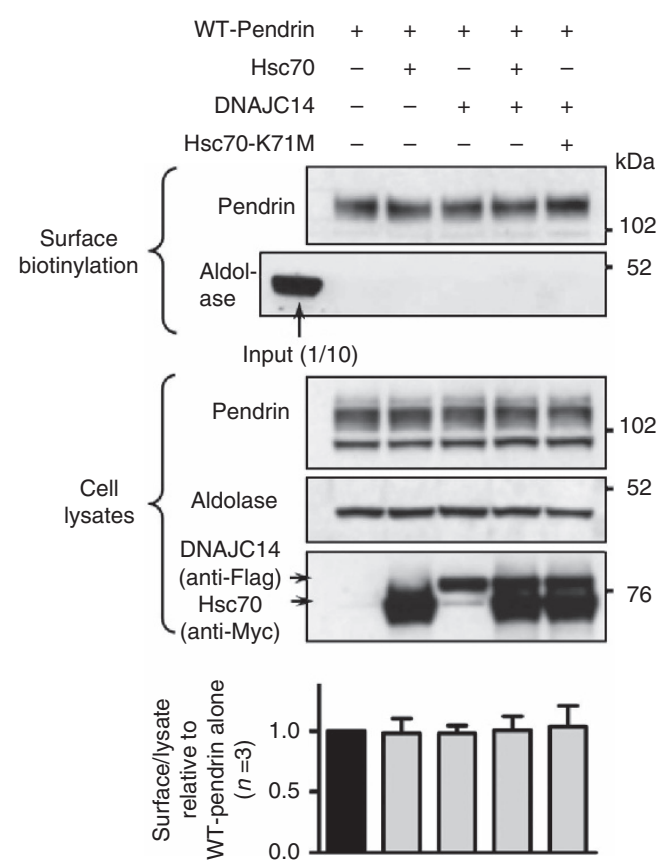

C
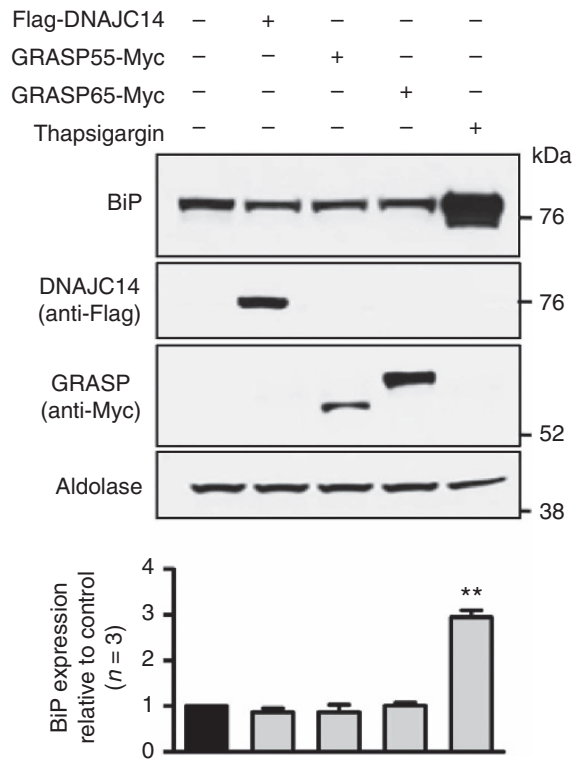

b

$\begin{array}{rcccccc}\text { H723R-Pendrin } & + & + & + & + & + & + \\ \text { Arf1-Q71L } & - & + & - & - & - & - \\ \text { Hsc70 } & - & - & + & - & + & - \\ \text { DNAJC14 } & - & - & - & + & + & + \\ \text { Hsc70-K71M } & - & - & - & - & - & +\end{array}$
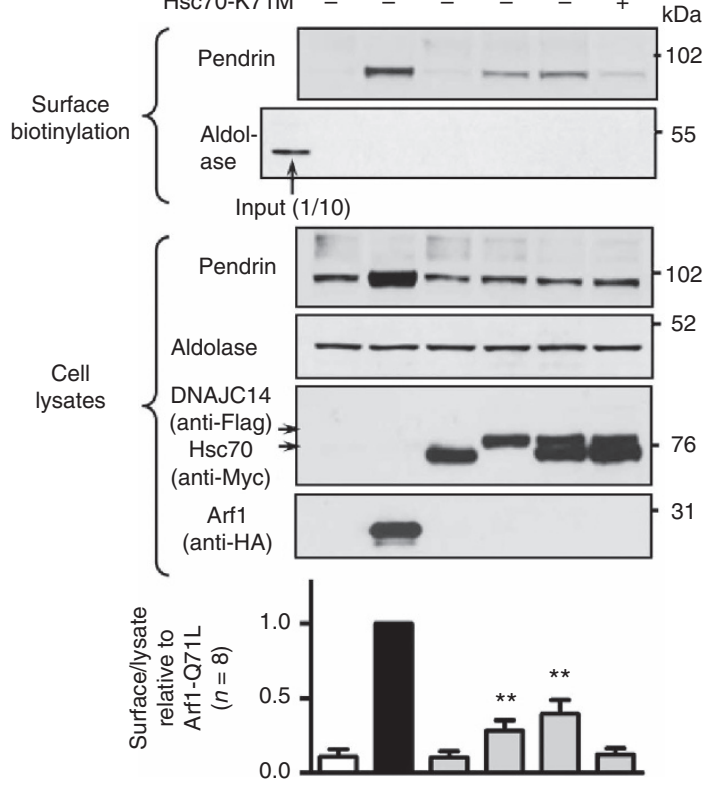

d
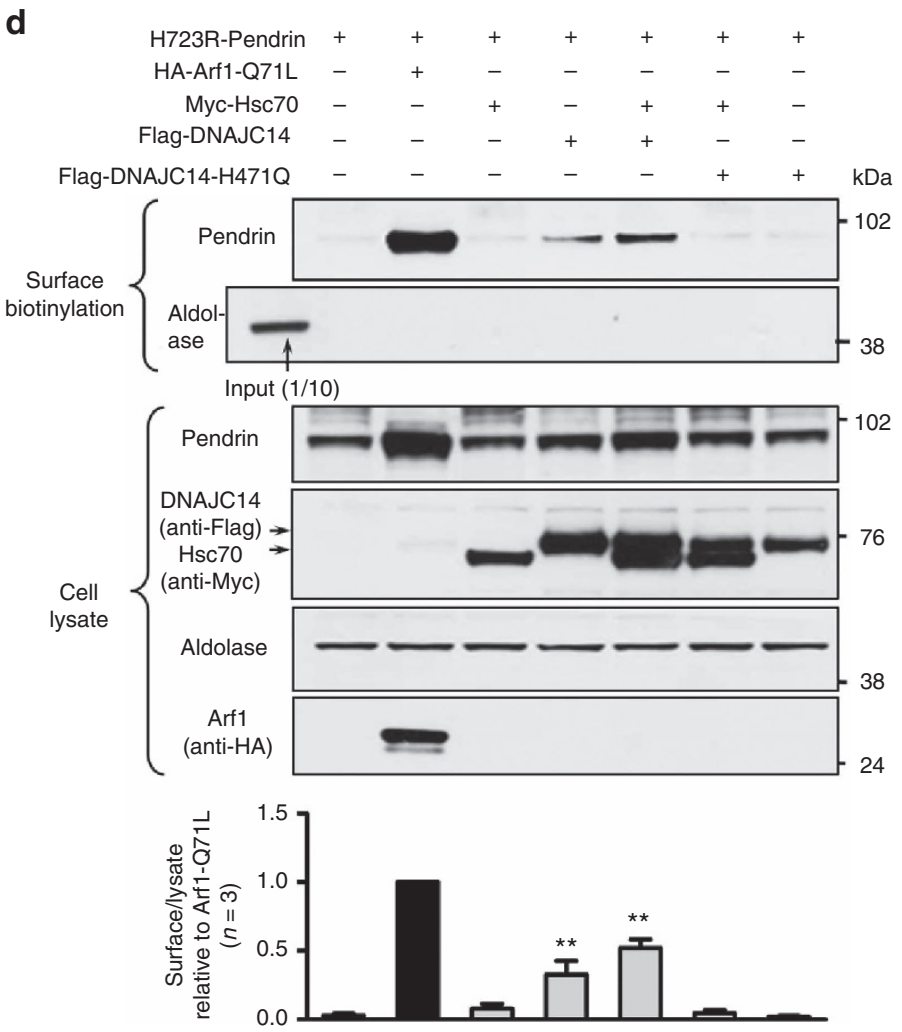

Figure 7 | DNAJC14 upregulation restores the cell-surface expression of H723R-pendrin. (a) The surface expression of WT-pendrin is not affected by upregulation of $\mathrm{Hsc70}$ and DNAJC14 or by inhibition of Hsc70 ATPase activity (Hsc70-K71M) in PANC-1 cells. (b) Overexpression of DNAJC14 alone or with Hsc70 induces the cell-surface expression of core-glycosylated H723R-pendrin in PANC-1 cells. Note that K71M abolished the effect of DNAJC14 to rescue H723R-pendrin at the surface. (c) Expression of BiP was analysed as an indicator of ER stress. Cells treated with thapsigargin were used as a positive control. Overexpression of DNAJC14, GRASP55 or GRASP65 did not increase BiP in PANC-1 cells. (d) The DNAJC14-H471Q mutation, which results in the loss of the Hsc70 ATPase accelerating activity, abolished the ability of DNAJC14 to rescue H723R-pendrin at the surface. Quantitation of multiple experiments is presented under each immunoblot. ${ }^{\star \star} P<0.01$ by one-way analysis of variance, difference from lane 1 , the number of replicates $(n)$ is presented in each panel. Unprocessed original scans of western blots are shown in Supplementary Fig. 10. 
a

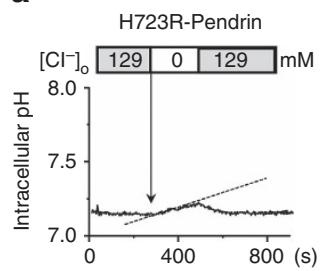

C
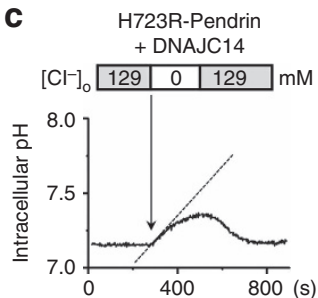

b H723R-Pendrin

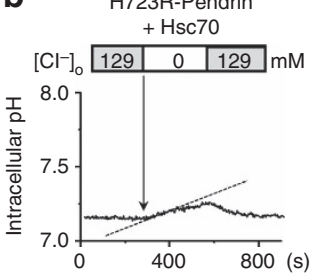

d

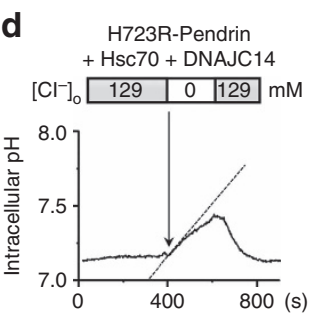

e

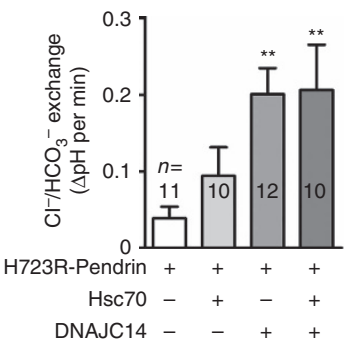

Figure 8 | Rescue of anion exchange activity of H723R-pendrin by DNAJC14. PANC-1 cells were transfected with the indicated plasmids and $\mathrm{Cl}^{-} / \mathrm{HCO}_{3}^{-}$ exchange activity was examined. Representative traces are shown in a-d, and a summary of multiple experiments is depicted in e. Overexpression of DNAJC14 alone, but not $\mathrm{Hsc70}$ alone, significantly increased the $\mathrm{Cl}^{-} / \mathrm{HCO}_{3}^{-}$exchange activity in $\mathrm{H} 723 \mathrm{R}$-pendrin-expressing PANC-1 cells. ${ }^{* \star} P<0.01$ by one-way analysis of variance, difference from H723R-pendrin alone (lane 1), the number of replicates ( $n$ ) is presented in each lane.

a Non-permeabilized

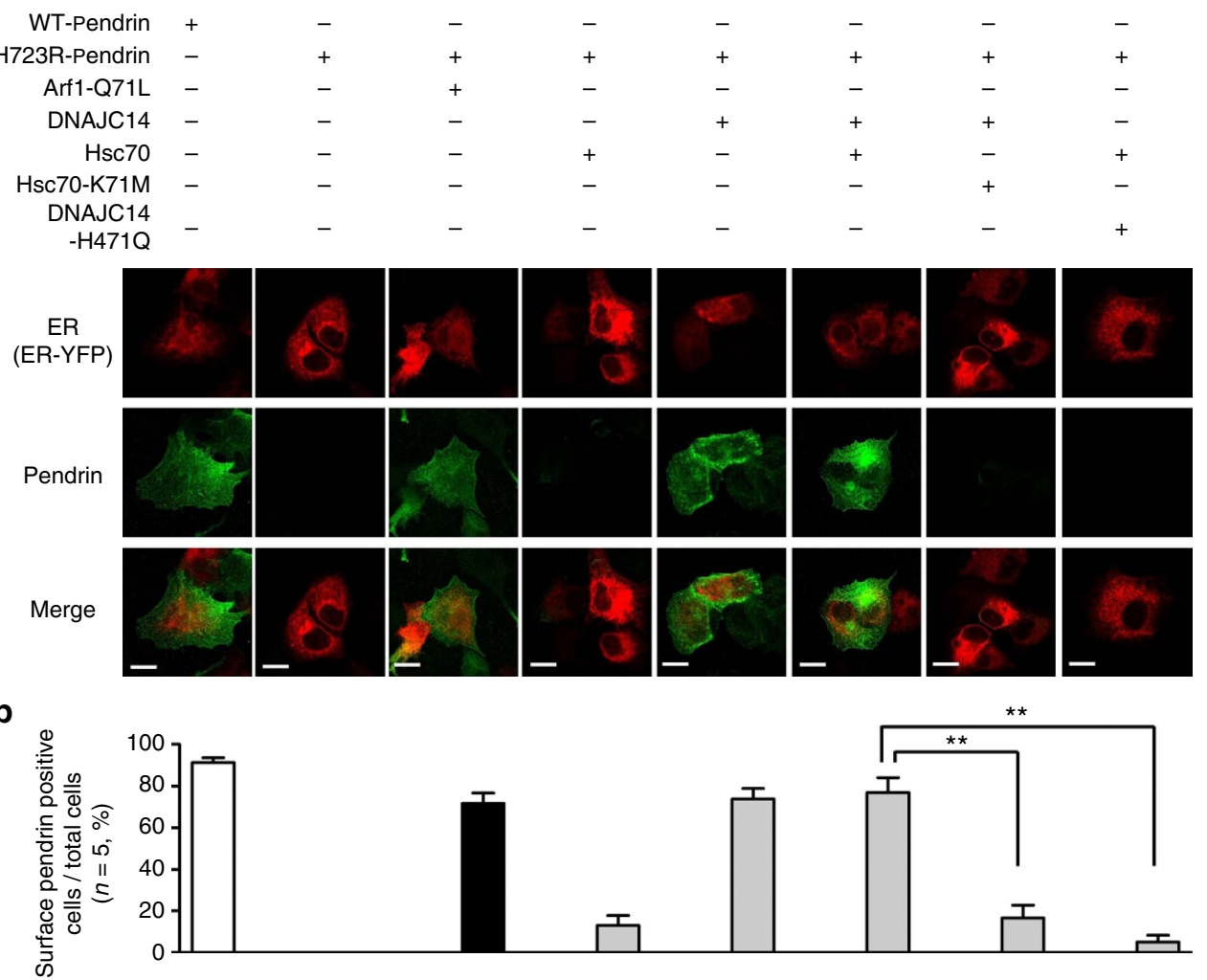

Figure 9 | DNAJC14 restores cell-surface expression of H723R-pendrin in immunostainings. The cell-surface expression of wild-type (WT) and H723R pendrins were examined in non-permeabilized PANC-1 cells. The ER marker protein ER-YFP was co-expressed. Representative images are shown in a. Quantitation of results from five independent experiments is shown in $\mathbf{b}$. Note that the Hsc70-K71M and DNAJC14-H471Q mutations strongly inhibited the ability of DNAJC14 to rescue H723R-pendrin at the cell surface. Scale bar, $20 \mu \mathrm{m}$. ${ }^{\star \star} P<0.01$ by one-way analysis of variance, $n=5$.

DNAJC14. In control mock-transfected PANC-1 cells, endogenous DNAJC14 was more evenly distributed in the cells and only a small fraction colocalized with Rab18 in perinuclear areas. Remarkably, ER-to-Golgi blockade by Arf1-Q71L (Fig. 10c) or Sar1-T39N (Fig. 10d) induced the movement of endogenous DNAJC14 into the punctate structures where Rab18 was also enriched (Fig. 10d,e).

\section{Discussion}

Most signal peptide-containing proteins, including transmembrane proteins, are synthesized in the ER and reach the plasma membrane by following the well-documented Golgi-mediated secretion pathway. However, recent evidence suggests that many cytosolic, nuclear, secretory and membrane proteins reach the cell surface by unconventional secretion pathways ${ }^{25}$. The unusual 
a

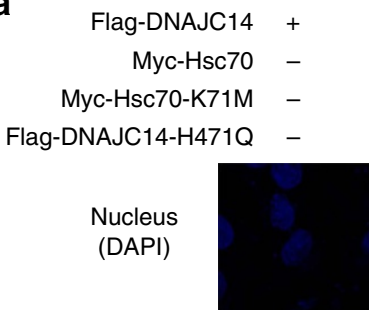

Hsc70

(anti-Myc)

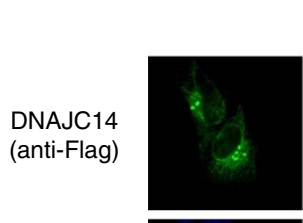

Merge

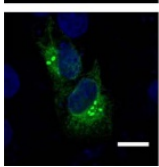

C

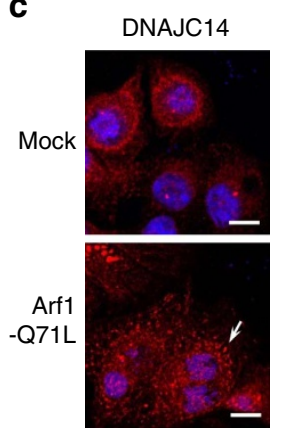

d

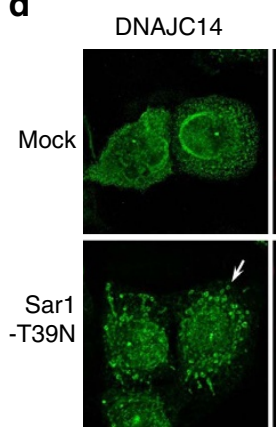

$+$

$+$

$-$

$-$
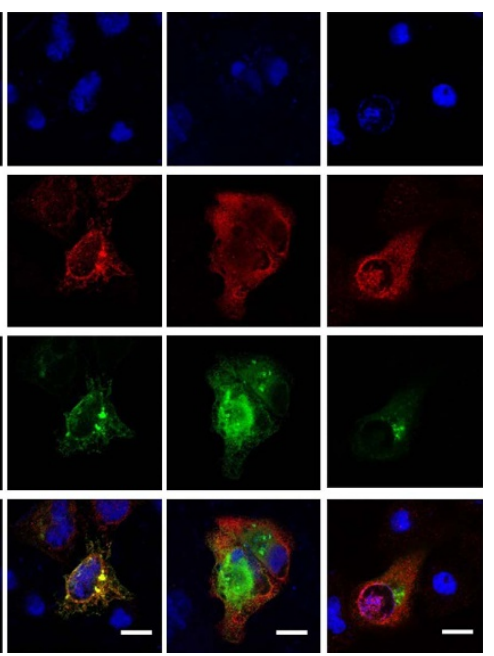

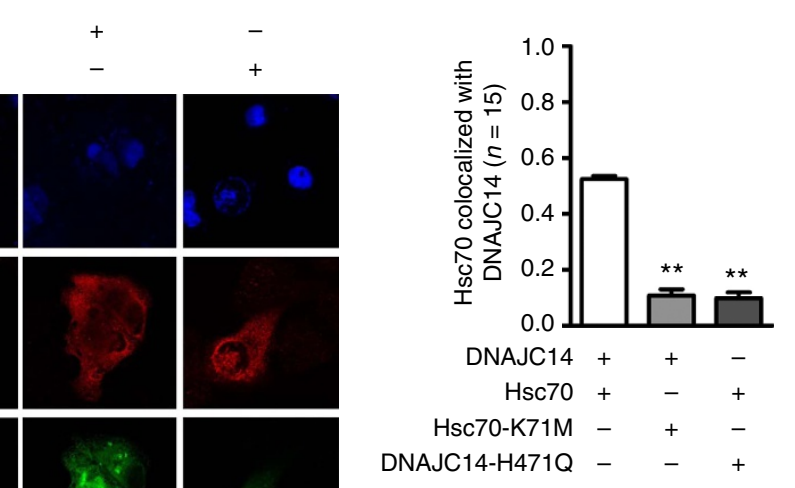

e

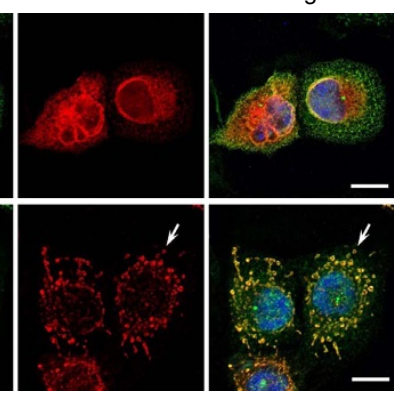

Figure 10 | Localization of DNAJC14 and Hsc70 in PANC-1 cells. (a,b) Subcellular localization of Flag-DNAJC14 and Myc-Hsc70 was examined in permeabilized PANC-1 cells. Representative images are shown in $\mathbf{a}$. Quantitation of results from multiple experiments is summarized in $\mathbf{b}$. A high degree of colocalization (an average of 52\%) was observed in cells stained doubly with Hsc70 and DNAJC14. The Hsc70-K71M and DNAJC14-H471Q mutations significantly reduced colocalization of Hsc70 and DNAJC14. Scale bar, $20 \mu \mathrm{m}$. ${ }^{\star \star} P<0.01$ by one-way analysis of variance, difference from lane $1, n=15$. (c-e) Endogenous DNAJC14 and Rab18 in PANC-1 cells were immunostained. ER-to-Golgi blockade by Arf1-Q71L (c) or Sar1-T39N (d) induced the movement of endogenous DNAJC14 into punctate structures and increased colocalization with Rab18 (d,e). Scale bar, $20 \mu \mathrm{m}$. ${ }^{\star \star} P<0.01$ by paired Student's $t$-test, $n=15$.

secretion events were first recognized by the release of cytoplasmic proteins that lack a signal peptide, such as FGF2 and interleukin $1 \beta$, into the extracellular space. Then, it was observed that signal peptide-containing proteins are also delivered to the plasma membrane via a route that bypasses the Golgi apparatus. In general, it is believed that most unconventional secretion events are not constitutive but stressinduced $^{26}$. Cellular stresses, such as inflammation, starvation and mechanical stress during development, induce unconventional secretion of cytoplasmic and membrane proteins. However, mechanisms and molecular components of these pathways are still not fully understood. In the present study, we provide evidence that $\mathrm{Hsc70}$ and DNAJC14 are involved in the unconventional secretion pathway induced by ER stress. Under normal conditions, transmembrane proteins that pass ERQC are delivered to the plasma membrane via the conventional Golgimediated route, and proteins that do not pass ERQC undergo ERAD. During ER stress or ER-to-Golgi blockade, unfolded protein response is activated to recruit, synthesize and cluster many ER and cytosolic chaperones necessary for the removal of accumulated protein in the ER. The activation of chaperone machinery in turn stimulates the activation of the unconventional secretion pathway, in addition to the well-established ERAD pathway, to relieve the protein burden in the ER. In this process, the ER co-chaperone DNAJC14, in cooperation with the cytosolic chaperone Hsc70, plays a critical role in targeting the transmembrane protein for unconventional secretion.

Hsc70 is a constitutively expressed molecular chaperone and achieves functional diversity based on specific cooperation with cochaperones. Hsc70 facilitates biogenesis and stabilization of CFTR in cooperation with Hdj1 (DNAJB1), Hdj2 (DNAJA1) and HOP (Hsp-organizing protein $)^{13,27,28}$, whereas it promotes protein ubiquitination and proteasomal degradation of misfolded CFTR when present with CHIP in eukaryotic cytosol ${ }^{14}$. In addition to protein folding and degradation, Hsc70 has been shown to be involved in the trafficking of proteins and small molecules, such as the uncoating of clathrin-coated pits, the recycling and endocytosis of synaptic vesicles for neurotransmitter release, and the maturation of steroid receptors ${ }^{29-32}$.

An important finding of the present study is the novel role of DNAJC14 in regulating the function of Hsc70. DNAJC14 was known to modulate the cell-surface trafficking of dopamine D1 receptor, the SNARE complex-mediated lysosomal trafficking, and the specific membrane targeting of yellow fever virus 
replication proteins ${ }^{24,33,34}$. However, the global role of DNAJC14 as a J domain-containing HSP70 co-chaperone remains elusive. The present results suggest that DNAJC14 switches the function of Hsc70 to divert the substrate protein (for example, H723Rpendrin) from proteasomal degradation to cell-surface secretion. DNAJC14 is an ER membrane protein, but both the $\mathrm{NH}_{2}$ and $\mathrm{COOH}$ termini and major functional domains, including the $\mathrm{J}$ domain, are exposed to the cytosol ${ }^{24}$. These structural characteristics imply that transmembrane proteins synthesized in the ER may be substrates of DNAJC14 during ER-to-Golgi blockade or ER stress conditions. The $\mathrm{H} 723$ residue of pendrin is located in the STAS (sulfate transporter and anti-sigma factor antagonist) domain of pendrin, which faces the cytosol. Under resting conditions, the cytosolic chaperone $\mathrm{Hsc70}$ and its cochaperones would censor the folding defects induced by the H723R mutation and bind to the misfolded pendrin to correct the folding defects. However, under conditions of DNAJC14 upregulation, such as during ER stress or in cells overexpressing exogenous DNAJC14, the J protein DNAJC14 may first bind to the client protein of folding-deficient H723Rpendrin, according to the canonical model of HSP70 chaperone machinery ${ }^{15}$, and bring it to the ATP-bound form of Hsc70. Then, the J domain-mediated stimulation of ATPase activity would convert $\mathrm{Hsc70}$ into an ADP-bound form, and this process would stabilize the interaction with the client cargo protein. As shown in Figs 4, 7, 9 and 10, the ATPase activity of Hsc70 is essential for the DNAJC14-mediated exodus of H723R-pendrin from ERAD to unconventional secretion. HSP70s invariably require a $J$ protein and, almost always, an NEF as partners ${ }^{15}$. While a specific J protein (DNAJC14) is required for the unconventional secretion of pendrin, depletion of any individual NEF was not critical for the cell-surface expression of H723R-pendrin induced by Arf1-Q71L (Fig. 5a) or by upregulation of DNAJC14 (Supplementary Fig. 6a,b). These results suggest that a redundancy exists among the functions of $\mathrm{NEF}$ isoforms and that they are not critically involved in the substrate specificity of Hsc70-mediated unconventional secretion.

Interestingly, conditions that induced unconventional cellsurface expression of CFTR and pendrin did not evoke unconventional secretion of the transferrin receptor (Supplementary Fig. 9), indicating that not all membrane proteins are unconventionally delivered to the cell surface during ER-toGolgi blockade or ER stress. Furthermore, although IRE1 and Hsc70 are commonly involved in the unconventional secretion of CFTR and pendrin (Supplementary Figs 1 and 3), there are also significant differences between the unconventional pathways of CFTR and pendrin (Fig. 1; Supplementary Figs 2 and 5). Taken together, these results suggest that a certain level of substrate specificity exists in ER stress-associated unconventional secretion, in particular, in the cargo recognition step (for example, CFTR by GRASPs and pendrin by DNAJC14). In the case of GRASP55, phosphorylation of its $C$ terminus by an IRE1-mediated signal is critical for association with the cargo protein CFTR ${ }^{17}$. Although the results in Fig. 5 indicate that a physical association with DNAJC14 underlies the Arf1-Q71L-induced unconventional secretion of pendrin, the precise molecular mechanism needs to be determined in future studies. It will be an intriguing task to investigate whether phosphorylation(s) at specific loci of DNAJC14 is required for ER-to-Golgi blockade-induced unconventional secretion of pendrin.

Blockade of the ER-to-Golgi transport induces an accumulation of many secretory and ER-resident proteins in the ER lumen ${ }^{17}$. This, in turn, will saturate the ERAD capacity of the cells and increase the total cellular levels of H723R-pendrin. In addition, activated DNAJC14 competes with other J proteins that would be involved in the degradation of misfolded proteins
(Supplementary Fig. 6c), which may further contribute to the increase in the total protein level of H723R-pendrin. In fact, the results in Fig. 1c-e indicate that Arf1-Q71L increased H723Rpendrin in cell lysates by an average of $136 \pm 29 \%(n=11)$. These findings may raise the possibility that accumulation of H723Rpendrin in the ER evokes nonspecific leakage of protein to the cell surface. However, several lines of evidence indicate that this is not the case and that a specific regulatory pathway is involved in the ER-to-Golgi blockade-induced cell-surface expression of H723Rpendrin. First, inhibition of $\mathrm{Hsc} 70$ or DNAJC14, which did not affect the cytosolic levels of H723R-pendrin, abolished the unconventional secretion of H723R-pendrin (Fig. 5b). Second, exogenous expression of DNAJC14, which did not increase cytosolic H723R-pendrin, evoked the cell-surface expression of H723R-pendrin (Fig. 7b). Last, all quantitative analyses of surface biotinylation assays using a ratio of surface/total pendrin indicated that ER-to-Golgi blockade or induction of ER stress prompted much higher cell-surface expression of coreglycosylated pendrin than its cytosolic increase, robustly suggesting the unconventional secretion of pendrin.

A remaining question is the nature of the vesicular traffic system that mediates the unconventional secretion of membrane protein cargos. The Rab18-containing vesicles seem to be responsible for the early secretion pathway immediately after ER exit as DNAJC14 was colocalized at these structures (Fig. 10c-e; Supplementary Fig. 8). Rab18 is the Ras-related small GTPase and is related to lipid droplet formation from the ER membrane ${ }^{35,36}$. Interestingly, the membrane protein caveolin1 has been shown to be redirected from the ER to lipid droplet vesicles under certain ER stress conditions ${ }^{37}$. Lipid droplets are, in general, composed of phospholipid monolayers. However, certain specific sites, such as 'wrinkles', would create ridges with local bilayer properties to which membrane proteins, such as class I major histocompatibility complex, could be anchored ${ }^{38}$. These findings suggest that lipid droplet vesicles might be involved in unconventional secretory transit of transmembrane proteins. However, there is also the possibility that vesicular traffics other than lipid droplets mediate the unconventional secretion. Future studies investigating the downstream trafficking machinery should aid in understanding the big picture of unconventional protein secretion.

Defects in protein folding can induce two types of diseases in human ${ }^{11}$. The first group is caused by the accumulation of aberrant proteins and its toxic effects, such as Huntington's, Parkinson's and Alzheimer's diseases. It is also plausible that disposal of misfolded proteins through the activation of unconventional secretion would be beneficial in treating patients with diseases caused by the toxic intracellular aggregation of misfolded proteins. The second group of diseases is characterized by the premature degradation of misfolded proteins, which eliminates the original function of the protein, such as in cystic fibrosis and Pendred syndrome. Although they have some defects in protein folding, many of these prematurely degraded membrane proteins, such as $\Delta$ F508-CFTR and H723Rpendrin, retain a certain level of their original activity when they reach the cell surface ${ }^{10,17,39}$. Therefore, many pharmacological and molecular efforts have been invested in approaches to facilitate the membrane targeting of these proteins. In this study, we showed that functionally active H723R-pendrin can be successfully transported to the epithelial cell surface by targeting it to unconventional protein secretion. The selective activation of this pathway may be a promising drug target for the treatment of patients with congenital deafness caused by foldingdeficient pendrin mutants. Considering that DFNB4 and Pendred syndrome are progressive until adolescence ${ }^{40,41}$, earlier diagnosis and correction would be beneficial for these patients. However, 
there would be some hurdles to overcome before a final implementation of this strategy in the clinic. For example, the cell-surface transported folding-deficient membrane proteins remain under the control of peripheral protein quality control, and thus, their retention time at the plasma membrane is relatively short ${ }^{42}$. A strategy combined with agents that inhibit peripheral quality control would be a measure to overcome this problem.

\section{Methods}

Cell culture and transfection. PANC-1 and HEK 293 cells were maintained in Dulbecco's modified Eagle medium-HG (Invitrogen, Carlsbad, CA, USA) supplemented with $10 \%$ fetal bovine serum and penicillin $\left(50 \mathrm{IU} \mathrm{ml}^{-1}\right) /$ streptomycin $\left(50 \mu \mathrm{g} \mathrm{ml}^{-1}\right)$. The mammalian expression plasmids for WT- and H723R-pendrin were subcloned into pcDNA3.1 $(+)$ (Invitrogen) and TAP expression vectors (Stratagene, La Jolla, CA, USA) from pCMV-myc-human pendrin described previously ${ }^{10}$, using the $\mathrm{KpnI}\left(5^{\prime}\right) / \operatorname{NotI}\left(3^{\prime}\right)$ and $\operatorname{BamHI}\left(5^{\prime}\right) / \mathrm{XhoI}\left(3^{\prime}\right)$ sites, respectively. Plasmids encoding human CFTR (WT-CFTR and $\triangle$ F508-CFTR), GRASP55, HASTX5, Myc-Sar1 and HA-Arf1 were described previously ${ }^{17}$. Human Hsc70, NckAP1, KIAA0980 and DNAJC14 clones were purchased from Open Biosystems (Lafayette, CO, USA) and subcloned into pCMV-myc or pCMV-Flag plasmids (Clontech, Palo Alto, CA, USA). Human Rab18-HA clone was purchased from Sino Biological (Beijing, China). pEYFP-ER was purchased from BD Biosciences (San Jose, CA, USA). Mutant plasmids were generated with a PCR-based sitedirected mutagenesis kit (Stratagene), according to the manufacturer's protocol. Oligonucleotide primer sequences for complementary DNA subcloning and mutagenesis are presented in Supplementary Table 2. A customized siRNA library (ON-TARGETplus as SMARTpool reagent) was purchased from Dharmacon (Lafayette). Detailed information for siRNAs used in this study is summarized in Supplementary Table 3. Plasmids or siRNAs were transiently transfected into the cells using Lipofectamine 2000 reagent (Invitrogen).

Chemicals and antibodies. Thapsigargin and BFA were purchased from SigmaAldrich (St Louis, MO, USA). Apoptozole was purchased from Millipore (Billerica, MA, USA). Anti-pendrin (G-19, epitope against C terminus), anti-HA, anti-Myc, anti-aldolase and anti-Flag antibodies were from Santa Cruz Biotechnology (Santa Cruz, CA, USA); anti-pendrin (ab66702, epitope against $\mathrm{N}$ terminus), anti-calnexin, anti-giantin, anti-Lamp1, anti-CHMP2B, anti-ATG7 and anti-Hsc70 were from Abcam (Cambridge, UK); anti-EEA1 was from BD Biosciences; anti-CD9 was from LSBio (Seattle, WA, USA); anti-DNAJC14 was from Novus (Littleton, CO, USA); anti-Rab7 was from Cell Signaling Technologies (Danvers, MA, USA); and anti-CFTR antibody (M3A7) was from Millipore. Detailed information for primary and secondary antibodies used in this study is summarized in Supplementary Table 4 .

Immunoprecipitation and surface biotinylation assay. For immunoprecipitation, cell lysates were mixed with the appropriate antibodies and incubated overnight at $4{ }^{\circ} \mathrm{C}$ in a lysis buffer containing $50 \mathrm{mM}$ Tris- $\mathrm{HCl}(\mathrm{pH} 8.0), 150 \mathrm{mM} \mathrm{NaCl}$, $1 \%(\mathrm{v} / \mathrm{v})$ Triton X-100, and complete protease inhibitor mixture (Roche Applied Science, Mannheim, Germany). Immune complexes were precipitated using mixed protein A/G beads (Pierce, Rockford, IL, USA) and then washed three times with lysis buffer before elution with $2 \times$ sodium dodecyl sulfate (SDS) sample buffer. Protein samples were separated by SDS-polyacrylamide gel electrophoresis (PAGE). The separated proteins were transferred to a nitrocellulose membrane and blotted with appropriate primary and secondary antibodies. Protein bands were detected by enhanced chemiluminescence, and the densities of the bands were measured using imaging software (Multi Gauge ver. 3.0; Fujifilm, Tokyo, Japan). For surface biotinylation assay, the surface proteins were biotinylated with sulfoNHS-SS-biotin (Pierce) for $30 \mathrm{~min}$ before cell lysis. Cells were washed with quenching buffer containing $1 \%$ bovine serum albumin (BSA) and then washed three times with phosphate-buffered saline (PBS). After cell lysis, the lysates were incubated overnight at $4{ }^{\circ} \mathrm{C}$ with $10 \%$ NeutrAvidin beads (Pierce). NeutrAvidinbound biotinylated proteins were centrifuged and washed three times with PBS and then eluted in $2 \times$ sample buffer. The next steps of the biotinylation assay were identical to those for the immunoblotting procedure described above. For the digestion of glycosylated pendrin by Peptide- $\mathrm{N}$-glycosidase F (PNGase F; New England Biolabs, Ipswich, MA, USA), protein samples were incubated with PNGase F (500 U per reaction) in solutions containing 1\% Triton X-100 for $2 \mathrm{~h}$.

TAP and identification of proteins by LC-MS/MS. TAP was performed using TAP expression vector with two tandem affinity tags, a streptavidin-binding peptide and a calmodulin-binding peptide (Stratagene). Cell lysates were applied to streptavidin resin, and unbound proteins were washed three times with streptavidin-binding buffer according to the manufacturer's protocol. Eluted protein complexes were applied to calmodulin resin and contaminants were removed by washing them three times with calmodulin-binding buffer. Tagged proteins and interacting partners were eluted with elution buffer, and eluted proteins were separated by SDS-PAGE. Protein bands of interest were excised and digested with trypsin using the following protocol ${ }^{43}$. Each protein band was excised from the PAGE gel, placed in a polypropylene tube, and briefly washed $4-5$ times with $150 \mu \mathrm{l}$ $1: 1$ acetonitrile $(\mathrm{ACN})$ per $25 \mathrm{mM}$ ammonium bicarbonate $(\mathrm{ABC}), \mathrm{pH}$ 7.8. The gel slices were dehydrated in $100 \% \mathrm{ACN}$ and then dried in a Speedvac concentrator. After reduction and alkylation, the gel slices were rehydrated in $30 \mu \mathrm{l} 25 \mathrm{mM} \mathrm{ABC}$ containing $20 \mu \mathrm{g}$ trypsin. After incubation at $37^{\circ} \mathrm{C}$ for $20 \mathrm{~h}$, the liquid was transferred to a clean tube. Tryptic peptides remaining in the gel matrix were extracted for $40 \mathrm{~min}$ at $30^{\circ} \mathrm{C}$ with $20 \mu \mathrm{l} 50 \%$ (v/v) aqueous ACN solution containing $0.1 \%(\mathrm{v} / \mathrm{v})$ formic acid. The combined supernatants were evaporated in the Speedvac and dissolved in $8 \mu \mathrm{l} 5 \%(\mathrm{v} / \mathrm{v})$ aqueous ACN containing $0.1 \%(\mathrm{v} / \mathrm{v})$ formic acid for mass spectrometric analysis. The resulting tryptic peptides were separated and analysed using a reverse phase capillary high-pressure liquid chromatography column directly coupled to a Finnigan LCQ ion-trap mass spectrometer (LC-MS/MS ${ }^{44}$. Both the $0.1 \times 20$-mm trapping and the $0.075 \times 130-\mathrm{mm}$ resolving columns were packed with Vydac $218 \mathrm{MS}$ low trifluoroacetic acid C18 beads ( $5 \mu \mathrm{m}$ diameter, $300 \AA$ pore size) and placed in-line. The peptides were bound to the trapping column for $10 \mathrm{~min}$ in $5 \%(\mathrm{v} / \mathrm{v})$ aqueous ACN containing $0.1 \%(\mathrm{v} / \mathrm{v})$ formic acid, and the bound peptides were eluted with a 50-min gradient of 5-80\% (v/v) ACN containing $0.1 \%(\mathrm{v} / \mathrm{v})$ formic acid at a flow rate of $0.2 \mu \mathrm{lmin}^{-1}$. For tandem mass spectrometry, the full mass scan range mode was an $\mathrm{m} / \mathrm{z}$ of $450-2,000 \mathrm{Da}$. After determination of the charge states of an ion using zoom scans, product ion spectra were acquired in MS/MS mode with a relative collision energy of $55 \%$. The individual MS/MS spectra were processed using the TurboSEQUEST software (Thermo Quest, San Jose, CA, USA). The generated peak list files were used to query either the MSDB or NCBI database using the MASCOT program (http://www.matrixscience.com). Modifications of methionine and cysteine (carbamidomethyl [C], deamidated [NQ], oxidation [M]) with a peptide mass tolerance of $2 \mathrm{Da}, \mathrm{MS} / \mathrm{MS}$ ion mass tolerance of $1 \mathrm{Da}$, allowance of missed cleavage of 1 , and charge states of $+1,+2$, and +3 , were all taken into account. Only significant hits as defined by MASCOT probability analysis were considered initially.

Immunocytochemistry. PANC-1 cells were cultured on coverslips and immunostained 1 day after transfection. Cells grown on coverslips were fixed and permeabilized by incubation in cold ethanol and acetone $(1: 1)$ for 8 min at $-20^{\circ} \mathrm{C}$. Nonspecific binding sites were blocked by incubation with $0.2 \mathrm{ml}$ PBS containing $5 \%$ horse serum, $1 \%$ BSA and $0.1 \%$ gelatin (blocking medium) for $1 \mathrm{~h}$ at room temperature. After the blocking step, cells were stained by incubating with appropriate primary antibodies and then treated with fluorophore-tagged secondary antibodies. Some cells were treated with primary and secondary antibodies after fixation in $4 \%$ formaldehyde without permeabilization to stain pendrin on the cell surface. Fluorescence images were obtained with a Zeiss LSM 780 confocal microscope (Carl Zeiss, Berlin, Germany). Morphometric analysis of the captured confocal images was performed using the MetaMorph microscopy analysis software (version 7.1; Molecular Devices, Sunnyvale, USA). For the quantification of the images under each condition, 24-bit confocal images containing red, green and blue components were converted into three 8-bit mono-channel images. The colocalization of DNAJC14 and each marker protein was analysed using the colocalization module of the MetaMorph Apps package. For the analyses of the surface expression of pendrin in non-permeabilized cells, pixels above the threshold level of 30 were defined as surface pendrin positive.

Quantitative real-time PCR. Quantitative real-time PCR (qPCR) was performed to examine the efficiency of siRNA treatments (Supplementary Fig. 3a) and to quantify DNAJC14 mRNA (Fig. 5b). qPCR primers for NckAP1 (Bioneer, Daejeon, Korea; catalogue no. P157442), Hsc70 (Bioneer, P219307), AP3B1 (Bioneer, P132135) and KIAA0980 (Bioneer, P257561) and a TaqMan probe for DNAJC14 (Applied Biosystems, catalogue no. Hs00372873_m1) were from commercial sources. Purified RNA samples from PANC-1 cells were reverse-transcribed using the iScript Select cDNA Synthesis Kit (Bio-Rad, Hercules, CA, USA). The total reaction volume was adjusted to $25 \mu \mathrm{l}$ with RNase-free water after mixing $500 \mathrm{ng}$ cDNA, $1 \mu \mathrm{l}$ primer (or $1 \mu \mathrm{l}$ TaqMan probe for DNAJC14) and $12.5 \mu \mathrm{l} 2 \times \mathrm{qPCR}$ SYBR Master Mix (Applied Biosystems, catalogue no. 4309155; or $12.5 \mu \mathrm{l} 2 \times$ TaqMan Gene Expression Master Mix for DNAJC14, Applied Biosystems, catalogue no. 4369016). Amplification was performed under the following cycling conditions: $95^{\circ} \mathrm{C}$ for $15 \mathrm{~min}$ followed by 40 cycles of $95^{\circ} \mathrm{C}$ for $15 \mathrm{~s}$ and $60^{\circ} \mathrm{C}$ for $40 \mathrm{~s}$. Triplicate analyses were performed for each cDNA. Relative mRNA expression levels were calculated via the comparative threshold cycle $\left(\mathrm{C}_{\mathrm{t}}\right)$ method with GAPDH as the control as follows: $\Delta \mathrm{C}_{\mathrm{t}}=\mathrm{C}_{\mathrm{t}}(\mathrm{GAPDH})-\mathrm{C}_{\mathrm{t}}$ (target gene). The foldchange in gene expression normalized to GAPDH and relative to the control sample was calculated as $2^{-} \Delta \Delta^{\mathrm{Ct}}$.

Measurement of $\mathrm{pH}_{\mathrm{i}}$ and $\mathrm{Cl}^{-} / \mathrm{HCO}_{3}^{-}$exchange activity. Measurements of $\mathrm{pH}_{\mathrm{i}}$ in HEK 293 and PANC-1 cells were performed with the $\mathrm{pH}$-sensitive fluorescent probe $2^{\prime}, 7^{\prime}$-bis-(2-carboxyethyl)-5-(and-6)-carboxyfluorescein (BCECF). After a 24-48-h transfection with plasmids encoding WT or H723R pendrin, cells were incubated with $2 \mu \mathrm{M} \mathrm{BCECF}$ acetoxy-methylester for $5 \mathrm{~min}$ and then perfused with a $\mathrm{HCO}_{3}^{-}$-buffered solution $\left(120 \mathrm{NaCl}, 5 \mathrm{KCl}, 1 \mathrm{MgCl}_{2}, 1 \mathrm{CaCl}_{2}, 10\right.$ D-glucose, 5 HEPES and $25 \mathrm{NaHCO}_{3}$ in $\mathrm{mmoll}^{-1}, \mathrm{pH}$ 7.4). BCECF fluorescence was recorded 
at the excitation wavelengths of 490 and $440 \mathrm{~nm}$ at a resolution of $2 / \mathrm{s}^{-1}$ on a recording setup (Delta Ram; PTI Inc., Edison, New Jersey, USA). $\mathrm{Cl}^{-} / \mathrm{HCO}_{3}^{-}$ exchange activities were estimated from the initial rate of $\mathrm{pH}_{\mathrm{i}}$ increase as a result of $\mathrm{Cl}^{-}$removal in the $\mathrm{HCO}_{3}^{-}$-containing buffer $\left(25 \mathrm{mM} \mathrm{HCO}_{3}^{-}\right.$with $\left.5 \% \mathrm{CO}_{2}\right) \cdot \mathrm{pH}_{\mathrm{i}}$ calibration was done with standard $\mathrm{pH}$ solutions containing $150 \mathrm{mM} \mathrm{KCl}$ and $5 \mu \mathrm{M}$ nigericin (Sigma-Aldrich). The intrinsic buffer capacity $\left(\beta_{\mathrm{i}}\right)$ was calculated by measuring $\Delta \mathrm{pH}_{\mathrm{i}}$ in response to $5-40 \mathrm{mM} \mathrm{NH}_{4} \mathrm{Cl}$ pulses in $\mathrm{Na}^{+}$-free solutions. Because the $\beta_{\mathrm{i}}$ values were not significantly affected by transfection with plasmids encoding WT-pendrin, H723R-pendrin, $\mathrm{Hsc70}$ or DNAJC14, the $\mathrm{Cl}^{-} / \mathrm{HCO}_{3}^{-}$ exchange activities were expressed as $\Delta \mathrm{pH}$ unit $\min ^{-1}$ without compensating for the buffer capacity.

\section{Measurement of $\mathrm{Cl}^{-} / \mathrm{I}^{-}$exchange activity of pendrin. $\mathrm{Cl}^{-} / \mathrm{I}^{-}$exchange} activity of pendrin was measured with the halide-sensitive fluorescent sensor yellow fluorescent protein (YFP)-H148Q/I152L/F46L (ref. 45). Fluorescence was detected using a FLUOstar fluorescence plate reader (BMG Labtech, Durham, North Carolina, USA) with excitation $(485 \pm 12 \mathrm{~nm})$ and emission $(>520 \mathrm{~nm})$ filters. Cells in a 96-well plate were washed three times with $150 \mathrm{mM} \mathrm{NaCl}$ solution, leaving $50 \mu \mathrm{l}$ in each well. The plate was transferred to a plate reader and the initial fluorescence was recorded for $20 \mathrm{~s}$ ( $400 \mathrm{~ms}$ per point). Then, $150 \mu \mathrm{l}$ of $150 \mathrm{mM} \mathrm{NaI}$ solution was added to each well. The $\mathrm{I}^{-}$influx mediated by the $\mathrm{Cl}^{-} / \mathrm{I}^{-}$exchange activity of pendrin quenched the fluorescence of YFP, and the slope of fluorescence change was analysed.

Statistical analysis. The results of the multiple experiments are presented as mean \pm s.e.m. Statistical analysis was performed using either Student's $t$-tests or an analysis of variance followed by the Newman-Keuls multiple comparison test, where appropriate. $P<0.05$ was considered statistically significant.

\section{References}

1. Mount, D. B. \& Romero, M. F. The SLC26 gene family of multifunctional anion exchangers. Pflugers Arch. 447, 710-721 (2004).

2. Park, H. J. et al. Genetic basis of hearing loss associated with enlarged vestibular aqueducts in Koreans. Clin. Genet. 67, 160-165 (2005).

3. Park, H. J. et al. Origins and frequencies of SLC26A4 (PDS) mutations in east and south Asians: global implications for the epidemiology of deafness. J. Med. Genet. 40, 242-248 (2003)

4. Tsukamoto, K. et al. Distribution and frequencies of PDS (SLC26A4) mutations in Pendred syndrome and nonsyndromic hearing loss associated with enlarged vestibular aqueduct: a unique spectrum of mutations in Japanese. Eur. J. Hum. Genet. 11, 916-922 (2003).

5. Wang, Q. J. et al. A distinct spectrum of SLC26A4 mutations in patients with enlarged vestibular aqueduct in China. Clin. Genet. 72, 245-254 (2007).

6. Wu, C. C., Yeh, T. H., Chen, P. J. \& Hsu, C. J. Prevalent SLC26A4 mutations in patients with enlarged vestibular aqueduct and/or Mondini dysplasia: a unique spectrum of mutations in Taiwan, including a frequent founder mutation. Laryngoscope 115, 1060-1064 (2005).

7. Dai, P. et al. Molecular etiology of hearing impairment in Inner Mongolia: mutations in SLC26A4 gene and relevant phenotype analysis. J. Transl. Med. 6, 74 (2008).

8. Dossena, S. et al. Functional characterization of wild-type and mutated pendrin (SLC26A4), the anion transporter involved in Pendred syndrome. J. Mol. Endocrinol. 43, 93-103 (2009).

9. Lee, H. et al. Correlation between genotype and phenotype in patients with bi-allelic SLC26A4 mutations. Clin. Genet. 86, 270-275 (2013).

10. Yoon, J. S. et al. Heterogeneity in the processing defect of SLC26A4 mutants. J. Med. Genet. 45, 411-419 (2008).

11. Vembar, S. S. \& Brodsky, J. L. One step at a time: endoplasmic reticulumassociated degradation. Nat. Rev. Mol. Cell Biol. 9, 944-957 (2008).

12. Strickland, E., Qu, B. H., Millen, L. \& Thomas, P. J. The molecular chaperone $\mathrm{Hsc70}$ assists the in vitro folding of the $\mathrm{N}$-terminal nucleotide-binding domain of the cystic fibrosis transmembrane conductance regulator. J. Biol. Chem. 272, 25421-25424 (1997)

13. Meacham, G. C. et al. The Hdj-2/Hsc70 chaperone pair facilitates early steps in CFTR biogenesis. EMBO J. 18, 1492-1505 (1999).

14. Meacham, G. C., Patterson, C., Zhang, W., Younger, J. M. \& Cyr, D. M. The Hsc70 co-chaperone CHIP targets immature CFTR for proteasomal degradation. Nat. Cell Biol. 3, 100-105 (2001).

15. Kampinga, H. H. \& Craig, E. A. The HSP70 chaperone machinery: J proteins as drivers of functional specificity. Nat. Rev. Mol. Cell Biol. 11, 579-592 (2010).

16. Young, J. C., Barral, J. M. \& Ulrich Hartl, F. More than folding: localized functions of cytosolic chaperones. Trends Biochem. Sci. 28, 541-547 (2003).

17. Gee, H. Y., Noh, S. H., Tang, B. L., Kim, K. H. \& Lee, M. G. Rescue of $\triangle$ F508-CFTR trafficking via a GRASP-dependent unconventional secretion pathway. Cell 146, 746-760 (2011).

18. Schroder, M. \& Kaufman, R. J. The mammalian unfolded protein response. Annu. Rev. Biochem. 74, 739-789 (2005).
19. Wiseman, R. L., Haynes, C. M. \& Ron, D. SnapShot: the unfolded protein response. Cell 140, 590-590.e2 (2010).

20. Rigaut, G. et al. A generic protein purification method for protein complex characterization and proteome exploration. Nat. Biotechnol. 17, 1030-1032 (1999).

21. Yi, Z. et al. Identification and characterization of the host protein DNAJC14 as a broadly active flavivirus replication modulator. PLoS Pathog. 7, e1001255 (2011).

22. Cho, H. J. et al. A small molecule that binds to an ATPase domain of Hsc70 promotes membrane trafficking of mutant cystic fibrosis transmembrane conductance regulator. J. Am. Chem. Soc. 133, 20267-20276 (2011).

23. Vos, M. J., Hageman, J., Carra, S. \& Kampinga, H. H. Structural and functional diversities between members of the human HSPB, HSPH, HSPA, and DNAJ chaperone families. Biochemistry 47, 7001-7011 (2008).

24. Yi, Z., Yuan, Z., Rice, C. M. \& MacDonald, M. R. Flavivirus replication complex assembly revealed by DNAJC14 functional mapping. J. Virol. 86, 11815-11832 (2012).

25. Nickel, W. \& Rabouille, C. Mechanisms of regulated unconventional protein secretion. Nat. Rev. Mol. Cell Biol. 10, 148-155 (2009).

26. Giuliani, F., Grieve, A. \& Rabouille, C. Unconventional secretion: a stress on GRASP. Curr. Opin. Cell Biol. 23, 498-504 (2011).

27. Young, J. C., Agashe, V. R., Siegers, K. \& Hartl, F. U. Pathways of chaperone-mediated protein folding in the cytosol. Nat. Rev. Mol. Cell Biol. 5, 781-791 (2004).

28. Farinha, C. M., Nogueira, P., Mendes, F., Penque, D. \& Amaral, M. D. The human DnaJ homologue (Hdj)-1/heat-shock protein (Hsp) 40 co-chaperone is required for the in vivo stabilization of the cystic fibrosis transmembrane conductance regulator by Hsp70. Biochem. J. 366, 797-806 (2002).

29. Augustine, G. J. et al. Clathrin and synaptic vesicle endocytosis: studies at the squid giant synapse. Biochem. Soc. Trans. 34, 68-72 (2006).

30. Bronk, P. et al. Drosophila Hsc70-4 is critical for neurotransmitter exocytosis in vivo. Neuron 30, 475-488 (2001).

31. Chappell, T. G. et al. Uncoating ATPase is a member of the 70 kilodalton family of stress proteins. Cell 45, 3-13 (1986).

32. Liu, T., Daniels, C. K. \& Cao, S. Comprehensive review on the HSC70 functions, interactions with related molecules and involvement in clinical diseases and therapeutic potential. Pharmacol. Ther. 136, 354-374 (2012).

33. Bermak, J. C., Li, M., Bullock, C. \& Zhou, Q. Y. Regulation of transport of the dopamine D1 receptor by a new membrane-associated ER protein. Nat. Cell Biol. 3, 492-498 (2001).

34. Tchernev, V. T. et al. The Chediak-Higashi protein interacts with SNARE complex and signal transduction proteins. Mol. Med. 8, 56-64 (2002).

35. Ozeki, S. et al. Rab18 localizes to lipid droplets and induces their close apposition to the endoplasmic reticulum-derived membrane. J. Cell Sci. 118, 2601-2611 (2005).

36. Martin, S., Driessen, K., Nixon, S. J., Zerial, M. \& Parton, R. G. Regulated localization of Rab18 to lipid droplets: effects of lipolytic stimulation and inhibition of lipid droplet catabolism. J. Biol. Chem. 280, 42325-42335 (2005).

37. Ostermeyer, A. G. et al. Accumulation of caveolin in the endoplasmic reticulum redirects the protein to lipid storage droplets. J. Cell. Biol. 152, 1071-1078 (2001)

38. Ploegh, H. L. A lipid-based model for the creation of an escape hatch from the endoplasmic reticulum. Nature 448, 435-438 (2007).

39. Denning, G. M. et al. Processing of mutant cystic fibrosis transmembrane conductance regulator is temperature-sensitive. Nature 358, 761-764 (1992).

40. Usami, S. et al. Non-syndromic hearing loss associated with enlarged vestibular aqueduct is caused by PDS mutations. Hum. Genet. 104, 188-192 (1999).

41. Cremers, C. W. et al. Progressive hearing loss, hypoplasia of the cochlea and widened vestibular aqueducts are very common features in Pendred's syndrome. Int. J. Pediatr. Otorhinolaryngol. 45, 113-123 (1998).

42. Okiyoneda, T. et al. Peripheral protein quality control removes unfolded CFTR from the plasma membrane. Science 329, 805-810 (2010).

43. Bahk, Y. Y. et al. Antigens secreted from Mycobacterium tuberculosis: identification by proteomics approach and test for diagnostic marker. Proteomics 4, 3299-3307 (2004).

44. Zuo, X. et al. Towards global analysis of mammalian proteomes using sample prefractionation prior to narrow $\mathrm{pH}$ range two-dimensional gels and using one-dimensional gels for insoluble and large proteins. Electrophoresis 22, 1603-1615 (2001).

45. Namkung, W., Yao, Z., Finkbeiner, W. E. \& Verkman, A. S. Small-molecule activators of TMEM16A, a calcium-activated chloride channel, stimulate epithelial chloride secretion and intestinal contraction. FASEB J. 25, 4048-4062 (2011).

\section{Acknowledgements}

We thank Dong Soo Chang for editorial assistance and the Yonsei-Carl Zeiss Advanced Imaging Center for technical assistance. This work was supported by grants 2013R1A3A2042197 and 2007-0056092 (to M.G.L), and 2015R1D1A1A02062202 (to J.J.) from the National Research Foundation, the Ministry of Science, ICT \& Future Planning, Republic of Korea. 


\section{Author contributions}

J.J., J.K., S.H.R. and I.J. performed the physiological and molecular experiments and analysed the data. R.S. performed the immunofluorescence experiments. H.Y.G. and J.Y.C. contributed to data analysis. J.J, J.K. and M.G.L. planned the study and contributed to analysis and interpretation of the data. All authors discussed the results and commented on the manuscript.

\section{Additional information}

Supplementary Information accompanies this paper at http://www.nature.com/ naturecommunications

Competing financial interests: The authors declare no competing financial interests.
Reprints and permission information is available online at http://npg.nature.com/ reprintsandpermissions/

How to cite this article: Jung, J. et al. The HSP70 co-chaperone DNAJC14 targets misfolded pendrin for unconventional protein secretion. Nat. Commun. 7:11386 doi: $10.1038 /$ ncomms11386 (2016).

(c) (i)

This work is licensed under a Creative Commons Attribution 4.0 International License. The images or other third party material in this article are included in the article's Creative Commons license, unless indicated otherwise in the credit line; if the material is not included under the Creative Commons license, users will need to obtain permission from the license holder to reproduce the material. To view a copy of this license, visit http://creativecommons.org/licenses/by/4.0/ 